\title{
Isolation of wheat bran-colonizing and metabolizing species from the human fecal microbiota
}

Kim De Paepe ${ }^{1}$, Joran Verspreet ${ }^{2,3}$, Mohammad Naser Rezaei ${ }^{3}$, Silvia Hidalgo Martinez ${ }^{4}$, Filip Meysman ${ }^{4,5}$, Davy Van de Walle ${ }^{6}$, Koen Dewettinck $^{6}$, Jeroen Raes ${ }^{7,8}$, Christophe Courtin ${ }^{3}$, Tom Van de Wiele ${ }^{\text {Corresp. } 1}$

1 Faculty of Bioscience Engineering, Department of Biotechnology, Center for Microbial Ecology and Technology (CMET), Universiteit Gent, Gent, Belgium

2 Flemish Institute for Technological Research (VITO), Mol, Belgium

3 Faculty of Bioscience Engineering, Leuven Food Science and Nutrition Research Centre (LFoRCe), Laboratory of Food Chemistry and Biochemistry, KU Leuven, Heverlee, Belgium

4 Faculty of Sciences, Department of Biology, Ecosystem Management Research Group (ECOBE), Universiteit Antwerpen, Antwerpen, Belgium

5 Department of Biotechnology, Delft University of Technology, Delft, The Netherlands

6 Faculty of Bioscience Engineering, Department of Food Technology, Safety and Health, Laboratory of Food Technology and Engineering (FTE), Universiteit Gent, Gent, Belgium

7 Department of Microbiology and Immunology, Rega Institute, KU Leuven, Leuven, Belgium

8 Center for Microbiology, VIB, Leuven, Belgium

Corresponding Author: Tom Van de Wiele Email address: Tom.VandeWiele@UGent.be

Undigestible, insoluble food particles, such as wheat bran, are important dietary constituents that serve as a fermentation substrate for the human gut microbiota. The first step in wheat bran fermentation involves the poorly studied solubilization of fibers from the complex insoluble wheat bran structure. Attachment of bacteria has been suggested to promote the efficient hydrolysis of insoluble substrates, but the mechanisms and drivers of this microbial attachment and colonization, as well as subsequent fermentation remain to be elucidated. We have previously shown that an individually dependent subset of gut bacteria is able to colonize the wheat bran residue. Here, we isolated these bran-attached microorganisms, which can then be used to gain mechanistic insights in future pure culture experiments. Four healthy fecal donors were screened to account for interindividual differences in gut microbiota composition. A combination of a direct plating and enrichment method resulted in the isolation of a phylogenetically diverse set of species, belonging to the Bacteroidetes, Firmicutes, Proteobacteria and Actinobacteria phyla. A comparison with 16S rRNA gene sequences that were found enriched on wheat bran particles in previous studies, however, showed that the isolates do not yet cover the entire diversity of wheat-bran colonizing species, comprising among others a broad range of Prevotella, Bacteroides and Clostridium cluster XIVa species. We, therefore, suggest several modifications to the experiment set-up to further expand the array of isolated species. 
1 Isolation of wheat bran-colonizing and metabolizing 2 species from the human fecal microbiota

3

4 Kim De Paepe ${ }^{1}$, Joran Verspreet ${ }^{2 *}$, Mohammad Naser Rezaei ${ }^{2}$, Silvia Hidalgo Martinez ${ }^{3}$, Filip 5 Meysman $^{3,4}$, Davy Van de Walle ${ }^{5}$, Koen Dewettinck ${ }^{5}$, Jeroen Raes ${ }^{6,7}$, Christophe M. Courtin ${ }^{2}$ and 6 Tom Van de Wiele ${ }^{1}$

$7 \quad{ }^{1}$ Center for Microbial Ecology and Technology (CMET), Department of Biotechnology, Faculty 8 of Bioscience Engineering, Universiteit Gent, Ghent, Belgium

$9 \quad{ }^{2}$ Laboratory of Food Chemistry and Biochemistry, Leuven Food Science and Nutrition Research 10 Centre (LFoRCe), Faculty of Bioscience Engineering, KU Leuven, Heverlee, Belgium

${ }^{3}$ Ecosystem Management Research Group (ECOBE), Department of Biology, Faculty of Sciences, Universiteit Antwerpen, Antwerp, Belgium

${ }^{4}$ Department of Biotechnology, Delft University of Technology, Delft, The Netherlands.

${ }^{5}$ Laboratory of Food Technology and Engineering (FTE), Department of Food Technology, Safety and Health, Faculty of Bioscience Engineering, Universiteit Gent, Ghent, Belgium

${ }^{6}$ Department of Microbiology and Immunology, Rega Institute, KU Leuven, Leuven, Belgium

${ }^{7}$ Center for Microbiology, VIB, Leuven, Belgium

* Current affiliation: Flemish Institute for Technological Research (VITO), Mol, Belgium

Corresponding Author:

Tom Van de Wiele ${ }^{1}$

Coupure Links 653, 9000 Ghent, Belgium

Tom.VandeWiele@UGent.be 


\section{ABSTRACT}

26

27

Undigestible, insoluble food particles, such as wheat bran, are important dietary constituents that serve as a fermentation substrate for the human gut microbiota. The first step in wheat bran fermentation involves the poorly studied solubilization of fibers from the complex insoluble wheat bran structure. Attachment of bacteria has been suggested to promote the efficient hydrolysis of insoluble substrates, but the mechanisms and drivers of this microbial attachment and colonization, as well as subsequent fermentation remain to be elucidated. We have previously shown that an individually dependent subset of gut bacteria is able to colonize the wheat bran residue. Here, we isolated these bran-attached microorganisms, which can then be used to gain mechanistic insights in future pure culture experiments. Four healthy fecal donors were screened to account for interindividual differences in gut microbiota composition. A combination of a direct plating and enrichment method resulted in the isolation of a phylogenetically diverse set of species, belonging to the Bacteroidetes, Firmicutes, Proteobacteria and Actinobacteria phyla. A comparison with 16S rRNA gene sequences that were found enriched on wheat bran particles in previous studies, however, showed that the isolates do not yet cover the entire diversity of wheat-bran colonizing species, comprising among others a broad range of Prevotella, Bacteroides and Clostridium cluster XIVa species. We, therefore, suggest several modifications to the experiment set-up to further expand the array of isolated species. 


\section{INTRODUCTION}

46

47 Gut microbiome research has recently started to focus on the microbial composition and functionality of distinct gut environments, such as the mucus layer (Belzer \& de Vos 2012; Bhat et al. 1980; Bollinger et al. 2007a; Bollinger et al. 2007b; Macfarlane \& Dillon 2007; Nava et al. 2011; Probert \& Gibson 2002; Swidsinski et al. 2008; Van den Abbeele et al. 2013; Van den Abbeele et al. 2012). The importance of undigested, insoluble food particles as microbial colonization sites and their impact on functionality, however, has been poorly studied (Macfarlane et al. 2011). Results obtained by Walker et al. (2008) and Macfarlane et al. (1997, 2006) are inconclusive with respect to the existence of a distinct microbial community associated with the particulate matter in fecal samples (Macfarlane \& Macfarlane 2006; Macfarlane et al. 1997; Walker et al. 2008). Leitch et al. (2007) previously pinpointed a specific colonization pattern of insoluble substrates in an anaerobic fermentor system, with wheat bran colonization being dominated by members of Clostridium cluster XIVa and Bacteroides species (Leitch et al. 2007). In an attempt to shed more light on the specific colonization of plant polysaccharides present in the human diet, we have previously performed a series of experiments using wheat bran as an insoluble model substrate. Static batch incubations and experiments in the Simulator of the Human Intestinal Microbial Ecosystem (SHIME) confirmed the colonization of wheat bran by a specific subset of gut bacteria, comprising Prevotella copri, Bacteroides ovatus/cellulosilyticus/stercoris/eggerthii/xylanisolvens, Roseburia faecis, Eubacterium rectale, Coprococcus eutactus, Hungatella hathewayi, Dialister succinatiphilus/propionicifaciens, Bifidobacterium faecale/adolescentis, Lactobacillus, Pediococcus, Fusobacterium and Enterobacteriaceae species (De Paepe et al. 2017; De Paepe et al. 2018). 
68 An essential step in resolving the driving force and mechanisms behind this specific substrate

69 attachment entails the study of pure cultures. To this end, species deposited in culture collections

70 can be used, offering the advantage of working with fully characterized bacteria, from which

71 genome information is available. Alternatively, bacteria can be isolated, permitting the discovery

72 of novel strains and ensuring the use of relevant strains (Greub 2012). Recent technological

73 advancements have revived interest in bacterial culturing. The so-called culturomics approach,

74 which involves high-throughput microbial culturing using different conditions and media, has

75 shown that a large fraction of the gut microbial community is culturable (Browne et al. 2016;

76 Hugon et al. 2015; Lagier et al. 2012; Lagier et al. 2016). While the automated picking and

77 identification of millions of colonies is a promising strategy to capture microbial diversity in the

78 gut, it is not a standard analysis that requires specialized equipment. As we are specifically

79 interested in a subset of bacteria, capable of colonizing and metabolizing the wheat bran residue,

80 we can considerably bring down our isolation efforts by targeted enrichment prior to isolation. To

81 this end, in the work presented here, the fecal microbial communities derived from four healthy

82 individuals were cultured using wheat bran as the sole nutrient source. The washed wheat bran

83 residue with the attached microbiota, was subcultured four times in fresh medium to selectively

84 enrich the wheat bran-colonizing and metabolizing species. Additionally, the same fecal samples

85 were directly plated on wheat bran agar, selecting only for wheat bran-metabolizing species. 


\section{8}

MATERIALS AND METHODS

89

90

91

92

93

94

95

96

97

98

101

102

103

104

105

106

107

108

109

110

111

Bacterial strains capable of metabolizing wheat bran as a sole nutrient source were isolated from a fecal slurry by a combination of direct plating and enrichment (Fig. 1). In order to account for inter-individual variability, the fecal sample of four different individuals was evaluated. Research incubation work with fecal microbiota from human origin was approved by the ethical committee of the Ghent University hospital under registration number B670201214538. Written informed consent was obtained from all participants. A fecal slurry was prepared according to De Boever et al. (2000) and consisted of a 20\% (w/v) fecal sample, suspended and homogenized in $0.1 \mathrm{M}$ phosphate buffer $\mathrm{pH}$ 6.8, supplemented with $1 \mathrm{~g} \mathrm{~L}^{-1}$ sodium thioglycolate (Sigma-Aldrich, St. Louis, MO, US), henceforth referred to as 'anaerobic phosphate buffer' (De Boever et al. 2000). All isolation work was subsequently carried out in an anaerobic workstation (GP-Campus, Jacomex, TCPS NV, Rotselaar, Belgium).

\section{Solutions and growth media}

All chemicals were purchased from Sigma-Aldrich (St. Louis, MO, US). The isolation medium used in the direct plating and enrichment method, contained pre-digested particle size reduced wheat bran $\left(50 \mathrm{~g} \mathrm{~L}^{-1}\right)$, with chemical composition determined as described in the supplementary information and displayed in Fig. 1. The medium was reinforced with vitamin and hemin stock solutions (Table S1 and S2) and buffered at pH 5.8 and 6.8 respectively, to mimic proximal and distal colon $\mathrm{pH}$ using a $0.1 \mathrm{M}$ phosphate buffer $\left(\mathrm{KH}_{2} \mathrm{PO}_{4} / \mathrm{Na}_{2} \mathrm{HPO}_{4}\right)(\mathrm{Cummings}$ et al. 1987; Duncan et al. 2009). The amount of wheat bran was reduced to $10 \mathrm{~g} \mathrm{~L}^{-1}$ for donors 3 and 4.

For the direct plating method, the particle size reduced wheat bran was incorporated in the buffered autoclaved molten agar (15 $\mathrm{g} \mathrm{L}^{-1}$; Thermo Fisher Scientific, San José, CA, US) medium prior to 
112 its solidification. Plates were poured in a laminar flow cabinet after addition of the filter sterilized

113 heat labile stock solution (Table S2) and the wheat bran and stored at $4^{\circ} \mathrm{C}$, for maximum two 114 weeks.

115 The enrichment was performed in a buffered liquid broth. Besides the vitamin solutions, resazurin

$116\left(1 \mathrm{mg} \mathrm{L}^{-1}\right)$ was added as a redox indicator. After verifying the $\mathrm{pH}$, the broth was heated with

117 intermittent shaking to remove dissolved oxygen until boiling, after which it was sparged with $\mathrm{N}_{2}$

118 gas (30 min) while the medium was cooling down in ice water. The medium was subsequently 119 dispensed in Hungate tubes (Glasgeratebau Ochs Gmbh, Bovenden-Lenglern, Finland) (8 mL per 120 tube) under a continuous gas flow and tubes were quickly sealed with butyl rubber stoppers and 121 aluminum crimp seals to limit oxygen exposure. The headspace was flushed with $\mathrm{N}_{2}$ for 30 cycles 122 using a gas exchange apparatus (Plugge 2005). The Hungate tubes were autoclaved. The filter 123 sterilized heat labile stock solution (Table S2) and wheat bran were added in the anaerobic 124 workstation, alongside $0.5 \mathrm{~mL}$ reducing reagent stock solution at the targeted $\mathrm{pH}$ (Table S3).

125 Purified, isolated bacterial colonies were further characterized in a defined general nutritional 126 YCFAG medium (Browne et al. 2016; Duncan et al. 2002). The medium was modified by adding $1270.1 \mathrm{M} \mathrm{KH}_{2} \mathrm{PO}_{4} / \mathrm{Na}_{2} \mathrm{HPO}_{4}$ and correcting the $\mathrm{pH}$ to 6.8 and 5.8 using $10 \mathrm{M} \mathrm{NaOH}$ and by replacing the cysteine- $\mathrm{HCl}$ and bicarbonate by the reducing reagent stock solution shown in Table S3. As 129 for the isolation medium, this reducing reagent stock solution was added to the YCFAG broth right 130 before use. The heat labile vitamins were added after autoclaving. YCFAG plates were poured in 131 the laminar flow cabinet and stored at $4^{\circ} \mathrm{C}$. Anaerobic YCFAG liquid broth was prepared by 132 boiling, sparging and flushing the medium as described before, except that $10 \mathrm{~mL}$ of the medium was distributed per tube. After subculturing to confirm purity, the obtained isolates in YCFAG medium were stored in cryovials at $-80^{\circ} \mathrm{C}$ in the presence of a cryoprotective agent (Table S4). 


\section{Direct plating}

137 In the anaerobic workstation, tenfold serial dilutions $\left(10^{-1}\right.$ to $\left.10^{-6}\right)$ of the $20 \%$ (w/v) fecal slurry 138 were prepared in $0.1 \mathrm{M}$ anaerobic phosphate buffer at $\mathrm{pH} 6.8$. The -3 to -6 dilutions were spread $139(100 \mu \mathrm{L})$ on the surface of a solid agar plate (both at $\mathrm{pH} 5.8$ and 6.8) containing wheat bran as the 140 sole nutrient source, using disposable sterile cell spreaders (VWR, Radnor, PA, US). Plates were 141 transferred to the anaerobic workstation at least $12 \mathrm{~h}$ before inoculation. After inoculation, plates 142 were incubated in the anaerobic workstation at $37^{\circ} \mathrm{C}$. To check for contamination, two plates, one 143 for each $\mathrm{pH}$, were inoculated with the anaerobic phosphate buffer used to prepare the serial 144 dilutions, without the addition of a bacterial mix derived from the fecal slurry. Plates were daily 145 inspected for growth. In case of perceivable growth, for each $\mathrm{pH}$, ten discrete colonies were picked 146 with an inoculating loop and streak plates were made on solid YCFAG agar medium. Plates were 147 incubated in the anaerobic workstation at $37^{\circ} \mathrm{C}$. Again, YCFAG plates without bacterial suspension were included as a control. Growth on the YCFAG plates was monitored and if pure

149 colonies were observed, a single colony was transferred to YCFAG broth in a Hungate tube, which was consequently incubated at $37^{\circ} \mathrm{C}$ on an orbital shaker $(90 \mathrm{rpm})$ in a $45^{\circ}$ tilted position outside of the anaerobic working station. When visual growth occurred, $1 \mathrm{~mL}$ suspension was sampled and stored at $-80^{\circ} \mathrm{C}$ in the presence of a cryoprotective agent $(1 \mathrm{~mL})$ (Table $\left.\mathrm{S} 4\right)$ and as a control to confirm purity, an inoculating loop of suspension was subcultured on YCFAG plates, to visually assess conformity of the colony appearance. 


\section{Enrichment}

157 In the anaerobic workstation (GP-Campus, Jacomex, TCPS NV, Rotselaar, Belgium), $2 \mathrm{~mL}$ of the

$15820 \%(\mathrm{w} / \mathrm{v})$ fecal slurry was inoculated in an enrichment tube, one for each $\mathrm{pH}$, containing $8 \mathrm{~mL}$

159 isolation medium with wheat bran as the sole nutrient source. Two tubes without fecal inoculum

160 were included as a control. The Hungate tubes were capped with butyl rubber stoppers and 161 aluminum crimp seals and removed from the anaerobic workstation for incubation at $37^{\circ} \mathrm{C}$ on an 162 orbital shaker $(90 \mathrm{rpm})$ in a $45^{\circ}$ tilted position. After $24 \mathrm{~h}$ the Hungate tubes, including the controls, 163 were transferred to the anaerobic workstation. The wheat bran residue was harvested on an 164 autoclaved filter paper inserted in an autoclaved glass funnel and rinsed three times with anaerobic 165 phosphate buffer to remove loosely attached luminal bacteria. The wheat bran residue was sampled 166 with a disposable inoculating loop and five (donors 1 and 2) or two (donors 3 and 4) loops were 167 transferred to a new Hungate tube with fresh isolation medium. This procedure was repeated three 168 times. The supernatant of the last transfer (referred to as 'luminal suspension') was serially diluted $169\left(10^{-1}\right.$ to $\left.10^{-6}\right)$ in anaerobic phosphate buffer in a 96-well plate and the -2 to -6 dilutions were plated onto solid YCFAG agar medium. Single colonies (10 per $\mathrm{pH})$ were isolated and pure cultures were

171 obtained as described for the direct plating method.

\section{Sampling and analysis}

174 The fecal slurry was aliquoted for the purpose of Short Chain Fatty Acids (SCFA) analysis and 175 DNA extraction, followed by next-generation 16S rRNA gene amplicon sequencing. Colonies on 176 the surface of the wheat bran containing solid agar plates were enumerated. The wheat bran residue 177 and liquid broth in the enrichment Hungate tubes were sampled after each transfer for DNA 178 extraction and SCFA analysis. Samples after the first and final enrichment from the low and high 
$179 \mathrm{pH}$ incubation for each donor were sent for next-generation 16S rRNA gene amplicon sequencing.

180 For one donor, the complete sequence of enrichments was analyzed. The pure cultures in the

181 YCFAG medium resulting from both approaches were identified by 16S rRNA gene Sanger

182 sequencing after DNA extraction and metabolically characterized by SCFA measurement. All data

183 visualization and processing was performed in R version 3.4.2 (2017-09-28) (R Core Team 2016),

184 unless stated otherwise. The R code is provided in Supplementary Data S1 and S2 under the form 185 of an Rmarkdown file and the knitted pdf version. Raw SCFA and 16S rRNA gene amplicon 186 sequencing data is included in the Supplementary data files S3-S9. 16S rRNA gene Sanger 187 sequences of the isolates are supplied as a compressed folder (Sanger_isolates.zip). Additionally, 188 DataS10-22 comprise i) user defined functions, ii) Mothur reports with the closest 16S rRNA gene 189 Sanger reference for each OTU obtained by $16 \mathrm{~S}$ rRNA gene amplicon sequencing, iii) OTU 190 sequences obtained by $16 \mathrm{~S}$ rRNA gene next-generation amplicon sequencing in fasta format and 191 iv) RDP taxonomic annotation of the $16 \mathrm{~S}$ rRNA gene Sanger sequences of the isolates, which are 192 all imported in the Rmarkdown file.

193 All samples for functional analysis and for DNA extraction (the pellet obtained after centrifuging 194 $250 \mu \mathrm{L}$ sample at $5000 \mathrm{~g}$ for $10 \mathrm{~min}$ or $0.250 \mathrm{~g}$ washed bran residue) were stored at $-20^{\circ} \mathrm{C}$. Samples for SCFA analysis of the enrichment tubes and fecal slurry were 1:2 diluted in demineralized water 196 prior to analysis.

SCFA analysis and a phenol-chloroform based DNA purification, following DNA extraction through chemical and mechanical lysis by multidirectional beating were performed according to De Paepe et al. (2017). The DNA quality was verified by electrophoresis on a $1.5 \%(\mathrm{w} / \mathrm{v})$ agarose gel and the DNA concentration was measured using the QuantiFluor ${ }^{\circledR}$ dsDNA kit (Promega, Madison, WI, US) and Glomax ${ }^{\circledR}-$ Multi+ system (Promega, Madison, WI, US). 
202 The 16S rRNA gene from the pure cultures was amplified by PCR with the 63F

203 (5'CAGGCCTAACACACATGCAAGTC3')

204 (5'CGGTGTGTACAAGGCCCGGGAACG3') primer pair in a BioRad T100'TM Thermal Cycler

205 (Applied Biosystems, Foster City, CA, US) (Lane 1991). Primers were synthesized by Biolegio

206 (Nijmegen, The Netherlands) and added in a final concentration of $0.2 \mu \mathrm{M}$ in sterile nuclease-free

207 water (Sigma-Aldrich, St. Louis, MO, US), containing $0.1 \mu \mathrm{L}$ Taq buffer $\mu \mathrm{L}^{-1}$ PCR-mix, 0.025

208 units Recombinant Taq DNA polymerase $\mu \mathrm{L}^{-1}$ PCR-mix, $0.2 \mu \mathrm{M}$ dNTP Mix, $1.5 \mu \mathrm{M} \mathrm{MgCl}_{2}$

209 (Fermentas Molecular Biology Tools, Waltham, MA, US), $0.75 \mu$ M BSA (Roche Applied Science,

210 Penzberg, Germany) and $0.04 \mu \mathrm{L}$ DNA extract $\mu \mathrm{L}^{-1}$ PCR-mix. The PCR amplification was

211 initiated by a pre-denaturation step $\left(5 \mathrm{~min}\right.$ at $\left.94^{\circ} \mathrm{C}\right)$, followed by repeated denaturation $(1 \mathrm{~min}$ at

$\left.21295^{\circ} \mathrm{C}\right)$, annealing $\left(1 \mathrm{~min}\right.$ at $\left.53^{\circ} \mathrm{C}\right)$ and extension $\left(2 \mathrm{~min}\right.$ at $\left.72^{\circ} \mathrm{C}\right)$ for $30 \mathrm{cycles}$, followed by $10 \mathrm{~min}$

213 at $72^{\circ} \mathrm{C}$. PCR-products were purified with the innuPREP PCRpure Kit (Analytik Jena, Jena,

214 Germany) and sent for molecular identification by bi-directional Sanger sequencing

215 (LGCGenomics, Teddington, Middlesex, UK). Forward and reverse 16S rRNA gene Sanger reads

216 were classified through the RDP web interface using the RDP SeqMatch tool, restricting the

217 database search to type strains with only near-full-length good quality sequences, and blasted in

218 NCBI against the 16S rRNA gene sequences, selecting only type material, with optimization of

219 the BLAST algorithm for highly similar sequences (accession date: June 2017) (Altschul et al.

220 1990; Cole et al. 2014; Wang et al. 2007). Results were manually compared and yielded a good

221 correspondence. Bioedit was used to assess sequence quality, by manual inspection of the sequence

222 traces in the chromatograms (Hall 1999). Short reads or reads with a lot of ambiguous base calls

223 were precluded from the analysis. 
224 Next-generation 16S rRNA gene amplicon sequencing of the V4 region (515F-806R) was 225 performed on an Illumina MiSeq platform (Illumina, Hayward, CA, US) using Illumina MiSeq v2

226

227

228

229

230

231

232

233

234

235

236

237

238

239

240

241

242

243

244

245

246 chemistry at the VIB Nucleomics core (VIB, Gasthuisberg Campus, Leuven, Belgium). Positive and negative controls were taken along as discussed in De Paepe et al. (2018).

The mothur software package (v.1.39.5) and guidelines were used to process the amplicon data as described in detail in De Paepe et al. (2018) (Kozich et al. 2013). An OTU is hereinafter defined as a collection of sequences with a length between 220 and 253 nucleotides that are found to be more than $97 \%$ similar to one another in the V4 region of their 16S rRNA gene after applying OptiClust clustering (Chen et al. 2013; Schloss \& Westcott 2011; Schloss et al. 2009; Wang et al. 2012). Taxonomy was assigned using the RDP version 16 and silva.nr_v123 database (Cole et al. 2014; Quast et al. 2013; Wang et al. 2007). The resulting OTU table and taxonomy file were loaded in R (R Core Team 2016). All samples from donor 4, except for the final enrichment step, were discarded due to an insufficient number of reads $(<100)$. The outcome of the enrichment procedure was assessed by computing richness (Chao1 Richness estimator) and diversity (Shannon, Simpson, inverse Simpson and Fisher alpha) estimators using vegan_2.4-4 (Oksanen et al. 2016). The proportional community composition was displayed in bar graphs. For this purpose, the OTU table was filtered according to the arbitrary cutoff's described by McMurdie and Holmes (2014), whereby OTUs observed in less than $5 \%$ of the samples and with read counts below 0.5 times the number of samples were removed (McMurdie \& Holmes 2014). At genus level RDP version 16 taxonomy is displayed. To arrive at a species level classification, OTUs were manually annotated using the RDP web interface using the RDP SeqMatch tool, restricting the database search to type strains with only near-full-length good quality sequences, and blasted in NCBI against the 16S rRNA gene sequences, selecting only type material, with optimization of the BLAST algorithm 
247 for highly similar sequences (accession date: June 2017) (Altschul et al., 1990; Wang et al.,

248 2007; Cole et al., 2014). Inconsistent species level taxonomy assignments were not reported. The

249 sequence data has been submitted to the NCBI database under accession number SRP091975.

250 Finally, the resulting OTUs were compared to the 16S rRNA gene Sanger reads of the obtained

251 isolates by means of a phylogenetic placement analysis. Forward and reverse Sanger sequences

252 for each donor were grouped into separate files and reverse complements and summary statistics

253 were obtained using the mothur software package (v.1.39.5) (Schloss et al. 2009). The 515F-806R

254 primer pair, used for Illumina MiSeq 16S rRNA gene amplicon sequencing was located in the

255 Sanger reads (forward and reverse compliment). In case both primers were not present on one and

256 the same read (either forward, or reverse), consensus sequences (contigs) were generated using the

257 sangeranalyseR package (version 0.1.0) (Lanfear 2015). Contigs with more than 100 degenerated

258 positions, indicative of a poor quality alignment, were omitted. A reference alignment was built

259 from the Sanger reads (for each donor separately) applying the sina alinger (Pruesse et al. 2012).

260 OTUs were aligned to this reference Sanger alignment in mothur (align.seqs), yielding a report

261 with the closest Sanger reference for each OTU based on kmer searching (Schloss et al. 2009).

262 This report was loaded into R ( $\mathrm{R}$ Core Team 2016). For each isolate in the report, the top two

263 OTUs with the highest SearchScores were selected. A fasta file was constructed containing these

264 OTUs. In order to compare the OTUs spanning the V4 region of the 16S rRNA gene with the near

265 full-length Sanger reads, the RAxML implementation of the evolutionary placement algorithm of

266 short reads, as introduced by Berger et al. (2011), was used (Stamatakis 2014). The bootstrap

267 supported maximum likelihood (ML) phylogenetic reference tree was also constructed using

268 RAxML, selecting the General Time Reversible model of nucleotide substitution under the

269 Gamma model of rate heterogeneity (GTRGAMMA) with the parsimony random seed set to 
270 12345. The rapid bootstrap analysis was conducted starting from $\mathrm{N}=1000$ distinct randomized

271 maximum parsimony trees and was followed by a search for the best-scoring ML tree with rapid

272 bootstrap random number seed 123 (Stamatakis 2014). The best scoring ML tree with the OTU

273 short read insertions was visualized in iTOL (Letunic \& Bork 2016). The proportional abundance

274 of the OTUs in the fecal slurry and in the luminal suspension after the last passage at $\mathrm{pH} 5.8$ and

2756.8 were integrated in the tree as a multi-value bar chart.

\section{SEM and cryo-SEM Visualization}

277 Native, pre-digested and fermented wheat bran samples were visualized using cryo-SEM and

278 desktop SEM. For the purpose of SEM microscopy, the bran samples were chemically dried with

279 hexamethyldisilazane (HMDS) as described by Araujo et al. (2003). After complete evaporation

280 of the HMDS, samples were mounted on an aluminum pin (diameter: $12 \mathrm{~mm}$ ) using double sided

281 carbon tape and subsequently gold sputtered for $45 \mathrm{sec}$ at $30 \mathrm{~mA}$ (Agar Sputter Coater B7340,

282 Agar Scientific, UK). Images were collected using a Phenom Pro X SEM microscope (Phenom-

283 World B. V., the Netherlands) with a beam intensity of $10 \mathrm{keV}$.

284 As an alternative to SEM microscopy, samples were also visualized via cryo-SEM using a Jeol

285 JSM 7100F scanning electron microscope (JEOL Ltd, Tokyo, Japan). A small amount of wheat

286 bran was placed on a sticky carbon surface mounted on an aluminium stub, vitrified in a nitrogen

287 slush and transferred under vacuum conditions into the cryo-preparation chamber (PP3010T

288 Cryo-SEM Preparation System, Quorum Technologies, UK) conditioned at $-140^{\circ} \mathrm{C}$.

289 Subsequently, the sample was sublimated for $20 \mathrm{~min}$ at $-70^{\circ} \mathrm{C}$ to remove frost artefacts, sputter-

290 coated with platinum using argon gas, transferred to the SEM stage at $-140^{\circ} \mathrm{C}$ and electron beam

291 targeted at $3 \mathrm{keV}$.

292 


\section{RESULTS \& DISCUSSION}

295

296 In an attempt to isolate human fecal bacteria capable of growing on and attaching to wheat bran,

297 two different approaches were adopted: direct plating on a wheat bran based solid agar medium

298 and plating the cultures after a series of enrichment steps. Inter-individual variability and $\mathrm{pH}$ were

299 previously shown to determine the outcome of wheat bran colonization (Cummings et al. 1987;

300 Duncan et al. 2009) and were accounted for in the present study by examining four different donors

301 at two $\mathrm{pH}$ values representative for proximal $(\mathrm{pH}$ 5.8) and distal $(\mathrm{pH}$ 6.8) colon conditions. As

302 wheat bran was not autoclaved to avoid structural modifications, a control wheat bran sample was

303 incubated under both $\mathrm{pH}$ conditions. Bacterial growth was observed in the control, despite the pre-

304 incubation at low $\mathrm{pH}$ in the presence of digestive enzymes, mimicking the gastro-intestinal transit.

$30516 \mathrm{~S}$ rRNA gene sequencing of the V4 region, however, revealed that one OTU accounted for $100 \%$

306 of the reads. This Enterobacteriaceae OTU could not be unambiguously classified and was not

307 recovered in any of the incubations inoculated with a fecal sample, nor did anything grow on the

308 YCFAG plates after enrichment of the control.

309 Enrichment of wheat bran-attached bacteria

310 Incubation of fecal microbiota with wheat bran as a sole nutrient source resulted in short chain

311 fatty acid (SCFA) production, concomitant with a marked acidification, confirming the microbial

312 growth that was visually observed as an increased optical density (Fig. 2). During the consecutive

313 enrichment steps, the microbial richness and diversity decreased, as illustrated in the case of donor

3141 (Fig. 3), confirming the effectiveness of the enrichment procedure. After the first $24 \mathrm{~h}$ incubation

315 of the fecal sample of donor 1 in the presence of wheat bran, the bran-attached community was

316 clearly enriched in Bifidobacterium OTU1 (most similar to B. faecale/adolescentis) at pH 5.8 and 
317 Dialister OTU12 at pH 6.8 (Fig. 4). This wheat bran residue served as an inoculum for the next 24

$318 \mathrm{~h}$ incubation, a procedure which was repeated three times in total. The enrichment of bifidobacteria

319 at low $\mathrm{pH}$ persisted during successive passages and lactobacilli appeared from the second

320 enrichment step onwards. Acetate was the main metabolic end product detected, but it must be

321 noted that lactate production was not measured. In the $\mathrm{pH} 6.8$ condition, butyrate was formed next

322 to acetate, which can be linked to the enrichment of Faecalibacterium prausnitzii OTU5 besides

323 Bifidobacterium OTU1. In the other two donors, next to Lactobacillus, bifidobacteria and $F$.

324 prausnitzii, an enrichment of Pediococcus, Enterobacteriaceae, Escherichia/Shigella and

325 Fusobacterium species was observed (Fig. 5 and 6). The successful colonization of bifidobacteria,

326 Lactobacillus and Pediococcus on wheat bran has been observed in the proximal colon

327 compartment of the Dietary Particle Mucus Simulator of the Human Intestinal Microbial

328 Ecosystem (DP-M-SHIME) (De Paepe et al. 2018). The efficient wheat bran colonization could

329 possibly be attributed to extensive adhesive properties and might involve the expression of pili or

330 the production of EPS (Foroni et al. 2011; Hidalgo-Cantabrana et al. 2014; Johansson et al. 2011;

331 Kankainen et al. 2009; Lebeer et al. 2012; Roos \& Jonsson 2002; Ruas-Madiedo et al. 2007; Van

332 den Abbeele et al. 2013; Van den Abbeele et al. 2009; Van den Abbeele et al. 2012). Their

333 preference for the proximal colon compartment was suggested to reflect their acid tolerance

334 (Chung et al. 2016; De Paepe et al. 2018; Duncan et al. 2009; Tannock 2004; Van den Abbeele et

335 al. 2012; Walker et al. 2005). In line with this, Lactobacillus and Pediococcus were confined to

336 the $\mathrm{pH} 5.8$ enrichments in donors 1 and 2, which were characterized by a large $\mathrm{pH}$ drop by more

337 than one $\mathrm{pH}$ unit (Fig. 2). In order to reduce the degree of acidification and associated enrichment

338 of $\mathrm{pH}$ tolerant species, the wheat bran concentration and seeding amount were lowered from 50 to

$33910 \mathrm{~g} \mathrm{~L}^{-1}$ and from five to two inoculating loops respectively in donors 3 and 4 . This resulted in a 
340 more moderate $\mathrm{pH}$ decrease to $6.36 \pm 0.15$ and $\mathrm{pH} 5.18 \pm 0.19$ and reduced the share of lactobacilli

341 in the enrichments. Donor 3 and 4, were instead characterized by large Escherichia/Shigella

342 proportions, both at $\mathrm{pH} 5.8$ and 6.8. Adhesive structures are well documented in

343 Enterobacteriaceae species and colonization of the wheat bran residue might bestow resilience to

344 a suboptimal lower pH (Duncan et al. 2009; Gaastra et al. 2014; Lawley \& Walker 2013; The et

345 al. 2016). Enterobacteriaceae were less present on the bran in the DP-M-SHIME and mostly

346 resided in the mucus layer. In the absence of a mucus micro-environment in static batch

347 incubations, however, we previously found Enterobacteriaceae to dominate the early stages of

348 wheat bran colonization (De Paepe et al. 2018, submitted to ISME Journal). The latter was also

349 observed for Fusobacteria, which were abundant in the final enrichment stage of donor 2 in the

350 present experiment. Finally, the establishment of $F$. prausnitzii in donors 1,3 and 4 is surprising

351 and was not perceived in any of the previous experiments. $F$. prausnitzii is positioned in the mucus

352 layer, close to the gut epithelium and is able to adhere to mucins, but not to epithelial cells (Khan

353 et al. 2012; Martin et al. 2017; Marzorati et al. 2014). Moreover F. prausnitzii was shown to adhere

354 to wheat bran in a synbiotic formulation (Khan et al. 2014). Little is known regarding its

355 mechanism of adhesion.

356 Besides the capacity to adhere, the enriched bacteria depended on wheat bran as the sole nutrient

357 source. In general, micro-organisms need a carbon source as building blocks for organic matter, a

358 nitrogen/phosphorous/sulphur source for protein and nucleic acid synthesis, some trace elements

359 such as iron, magnesium, cobalt and manganese as cofactors for enzymes, and, in case of

360 auxotrophic growth, some vitamins and amino acids (Madigan \& Brock 2011). Wheat bran is a

361 versatile substrate, containing all of the above compounds (Hemdane et al. 2016). The compounds

362 are, however, part of a complex macro-molecular configuration. Carbohydrates in wheat bran 
363 mainly consist of non-starch cell wall polysaccharides (NSP), comprising arabinoxylans, $\beta$-glucan,

364 cellulose and fructan, which are physically intertwined with lignin (Hemdane et al. 2016). Some

365 residual starch can be present in the attached endosperm resulting from the crude milling process

366 (Fig. 7-8) (Hemdane et al. 2016; United States Department of Agriculture (USDA) 2016). The

367 residual endosperm fraction also contains some proteins (Chick et al. 1947; Shewry 2009). The

368 major part of the endosperm components is expected to disappear during a pre-digestion with

369 gastric and pancreatic enzymes (Fig. 7) (Amrein et al. 2003; Chick et al. 1947). Wheat bran

370 proteins, however, are also located in aleurone cells, which are more recalcitrant to digestion

371 (Amrein et al. 2003; Arte et al. 2015). This complex wheat bran structure and its insoluble nature

372 limits fermentability. In that sense, the retrieved enriched bacteria are unexpected as none of the

373 species is considered to be a wheat bran primary degrader, judged by the fact that they are not

374 equipped with the enzymatic complement required to solubilize and degrade cellulose and

375 arabinoxylan polymers.

376 Bifidobacteria generally prefer arabinoxylan oligosaccharides (AXOS) as a substrate (van den

377 Broek et al. 2008). Bifidobacterium longum and Bifidobacterium adolescentis, however, are able

378 to grow on arabinoxylans (Savard \& Roy 2009; van den Broek et al. 2008). Genomic studies

379 confirmed the presence of arabinofuranosidases and xylosidases in these species. Bifidobacterium

380 longum also possesses a multi-domain enzyme with a putative endo-xylanase (GH43) flanked by

381 two carbohydrate-binding modules (CBM) that might interact with xylans. (van den Broek et al.

382 2008). Arabinoxylan or AXOS consumption by lactic acid bacteria (LAB) received less attention,

383 so far (Michlmayr et al. 2013). Lactobacilli have been reported to respond to AXOS treatment in

384 the SHIME model but Lactobacillus brevis is the only species for which arabinofuranosidase and

385 xylosidase activity is evidenced (Michlmayr et al. 2013; Sanchez et al. 2009). Based on functional 
386 predictions, it is suggested that the AXOS degrading capacity might be shared by Pediococcus and 387 Enterococcus spp. (Michlmayr et al. 2013). Interestingly, despite this apparently limited wheat 388 bran degrading capacity, our findings are consistent with Enterococcus spp., Lactobacillus spp. 389 and Pediococcus pentosaceus being the main species recovered from spontaneous bran 390 fermentations and the fact that wheat bran is often used as a substrate in industrial fermentations 391 with lactobacilli (Arte et al. 2015; Katina et al. 2012; Prückler et al. 2015). In this context, native 392 wheat bran is used, containing more residual starch. Moreover, the activity of endogenous wheat 393 bran endoxylanases and cinnamoyl esterases, solubilizes the arabinoxylans and increases the 394 availability of arabinose and xylose monomers, which can be used by lactobacilli (Katina et al. 395 2012; Prückler et al. 2015). Besides endogenous xylanases, wheat kernels can contain xylanases 396 from microbial origin (Dornez et al. 2006). The fate of these wheat bran associated xylanases and 397 other endogenous wheat bran polymer degrading enzymes during in vivo gastro-intestinal digestion is unknown. But, a reduced, yet, preserved activity of those enzymes has been observed 399 after in vitro pre-digestion (unpublished results De Paepe et al.), which could explain the isolation 400 of species lacking these first-line enzymes. Enterobacteriaceae and Fusobacterium species are 401 capable of saccharolytic fermentation, but are also lacking xylan and cellulose degrading enzymes 402 (Brady et al. 2009; Cantarel et al. 2009; Gao et al. 2015; Lombard et al. 2014; Marzorati et al. 403 2017; Mazur \& Zimmer 2011; Richardson et al. 2013; Robrish et al. 1991; Salamanca-Cardona et 404 al. 2014; Thompson et al. 1997). F. prausnitzii is shown to degrade wheat bran in pure cultures to 405 a small extent ( $\sim 4 \%$ substrate loss) (Duncan et al. 2016). The required enzymatic activity is not 406 experimentally characterized but genomic predictions have identified several glycosyl hydrolases which can display $\beta$-xylosidase (EC 3.2.1.37), $\alpha$-L-arabinofuranosidase (EC 3.2.1.55), endo-1,4408 $\beta$-xylanase (EC 3.2.1.8) and feruloyl esterase (EC 3.1.1.73 ) activity 
409 (GH1,GH3,GH31,GH43,CE1), as well as a possible xylan binding domain (CBM13) (Cantarel et 410 al. 2009; Lombard et al. 2014). The release of ferulic acid from wheat bran by a pure culture of $F$.

411 prausnitzii has been demonstrated. Additionally, $F$. prausnitzii can benefit from the high acetate 412 concentrations through cross-feeding, supporting our finding of $F$. prausnitzii to be one of the 413 isolates (Duncan et al. 2002; Rios-Covian et al. 2015).

414 Besides the carbohydrate degrading capacity, growth on wheat bran as the sole nutrient source 415 requires proteolytic activity. The breakdown of proteins is essential for the assimilation of peptides 416 and amino-acids into new microbial biomass and can sustain fermentation leading to the 417 production of branched SCFA and ammonium (Macfarlane et al. 1986). Ammonium was not 418 measured in this study, and branched SCFA concentrations were negligible, despite the proteolytic 419 fermentation capacity of some of the enriched species (Enterobacteriaceae, Fusobacterium) 420 (Marzorati et al. 2017; Richardson et al. 2013; Robrish et al. 1991). The breakdown of wheat bran 421 proteins by gut bacteria is poorly studied. Wheat bran fermentation by LAB starter cultures has 422 indicated that enzymes from microbial origin contribute to the size reduction of oligopeptides and 423 the generation of free amino acids, while the release of oligopeptides depended on the endogenous 424 wheat bran proteases (Arte et al. 2015). Isolation experiments with gluten proteins revealed that 425 Enterococcus, Bifidobacterium, Pediococcus, Lactobacillus and Bacteroides fragilis are capable 426 of hydrolyzing gluten proteins and derived peptides (Caminero et al. 2014). Interestingly, $F$. 427 prausnitzii and Bacteroides dorei were recovered from liquid incubations with gluten proteins as 428 the main nitrogen source, but could not be isolated from solid agar media (Caminero et al. 2014).

\section{Isolation of wheat bran degrading and attached bacteria}

430 The luminal suspension obtained after the final enrichment step was plated onto nutritional 431 medium and alternatively, the fecal sample was directly plated on wheat bran based solid agar 
432 plates. Control wheat bran agar plates, which were inoculated with anaerobic phosphate buffer,

433 without the addition of a fecal sample showed no growth. From both strategies, at pH 5.8 and 6.8,

434 ten single colony isolates were picked. Isolates were identified through 16S rRNA gene Sanger

435 sequencing (Table 1).

436 Plating of the enrichment suspension resulted in the isolation of Lactobacillus and Collinsella

437 species in donor 1, Escherichia/Shigella, Pediococcus, Bifidobacterium and Enterococcus species

438 in donor 2, Escherichia/Shigella, Enterococcus and Klebsiella species in donor 3 and Collinsella,

439 Escherichia/Shigella, Eubacterium, Hungatella and Bifidobacterium species in donor 4 (Fig. S1-

$440 \mathrm{~S} 4$, Table 1). The isolated species after enrichment correspond to the microbial community

441 composition determined by Illumina sequencing, with the exception of $F$. prausnitzii and

442 Fusobacterium mortiferum, which were enriched but could not be isolated on solid agar plates. $F$.

443 prausnitzii is difficult to isolate due to its oxygen sensitivity (Duncan et al. 2002; Khan et al. 2014;

444 Lopez-Siles et al. 2017). All isolation work was carried out in an anaerobic workstation and the

445 YCFAG medium has been shown to support $F$. prausnitzii growth (Duncan et al. 2002; Khan et

446 al. 2012). The solid agar media were, however, not pre-reduced in this experiment, as opposed to

447 the liquid broth, which explains why $F$. prausnitzii could not be cultivated on YCFAG plates

448 (Holdeman et al. 1977). The direct plating method resulted in the additional isolation of

449 Bacteroides, Bifidobacterium and Streptococcus species (Table 1). This is in agreement with the

450 results from the enrichment series of donor 2, showing a higher diversity, including Bacteroides

451 species, after the first $24 \mathrm{~h}$ of incubation.

\section{Discussion of the experimental set-up}

453 The enrichment and isolation of predominantly LAB, bifidobacteria and Enterobacteriaceae at

454 first sight seems to contest the previous reports of wheat bran colonization by a subset of 
455 Prevotella, Bacteroides and Clostridium cluster XIVa organisms (De Paepe et al. 2017; Duncan

456 et al. 2016; Leitch et al. 2007). There are, however, some important differences in experimental

457 set-up that might account for the observed disparity and need to be addressed in future isolation

458 procedures.

459 First of all, the comparable enrichment of LAB, bifidobacteria, Streptococci and 460 Faecalibacterium, in the current study and in an isolation experiment using gluten as the major 461 protein source, could indicate an inability of Prevotella, Bacteroides and Clostridium species to 462 compete for wheat bran proteins. This would explain their limited growth compared to previous 463 studies using a protein-rich medium containing peptone and yeast-extract (De Paepe et al. 2017; 464 De Paepe et al. 2018).

465 Secondly, the $24 \mathrm{~h}$ incubation period in between transfers in the present study might have 466 influenced the results. A detailed analysis of the time course of wheat bran colonization and 467 fermentation. (Submitted to ISME Journal) revealed a succession of bacterial taxa alternately 468 dominating the community over a $72 \mathrm{~h}$ timespan. Early stages were dominated by 469 Enterobacteriaceae and Fusobacterium species and characterized by a low butyrate production.

470 After $48 \mathrm{~h}$, the butyrate ratio increased, corresponding to donor-dependent proportional increases 471 of Bacteroides ovatus/stercoris, Prevotella copri and Firmicutes species. We hypothesized that 472 depletion of the easily digestible compounds induced a shift towards carbohydrate degrading 473 specialists, possessing the enzymatic capacity to breakdown the complex molecules. This 474 hypothesis is also valid with wheat bran as the sole nutrient source. Apart from residual starch, the 475 more fermentable wheat bran components are located in the aleurone layer (Amrein et al. 2003; 476 Stevens \& Selvendran 1988). Indeed, we observed a preferential microbial colonization and 477 degradation of aleurone through SEM (Fig. 8). This aleurone degradation might be aided by the 
478 activity of wheat kernel associated endogenous or microbial enzymes, including xylanases. The

479 latter remains speculative, as these enzymes might be deactivated upon gastro-intestinal passage

480 and their activity can be inhibited by TAXI (Triticum aestivum xylanase inhibitor) and XIP

481 (xylanase-inhibiting protein) xylanase inhibitors, which are also present in wheat bran.

482 Finally, the large extent of acidification due to the high wheat bran concentration in the first two 483 donors $\left(50 \mathrm{~g} \mathrm{~L}^{-1}\right)$ might be responsible for the unintentional selection for acidophilic or aciduric 484 LAB.

485 In order to investigate the above hypotheses, it would be interesting to repeat the enrichment 486 procedure with a medium containing an additional protein source, extending the incubation time 487 in between passages to $48 \mathrm{~h}$ and ensuring a sufficient buffering capacity. This might result in the 488 enrichment and isolation of a vastly different array of gut bacteria. In addition, for the direct plating 489 strategy, the medium should be pre-reduced.

490

491

492

494

495

496

497

498

499

500

\section{CONCLUSIONS}

Enrichment of the wheat bran-colonizing microbial community resulted in the isolation of a diverse set of Lactobacillus, Bifidobacterium, Collinsella, Escherichia/Shigella, Pediococcus, Enterococcus, Klebsiella, Eubacterium and Hungatella species. These isolated species were also found to be enriched on the wheat bran residue by next-generation amplicon sequencing, demonstrating that the proposed enrichment procedure is a sensible and efficient approach to isolate wheat bran-colonizing species. As insoluble wheat bran presented the sole carbohydrate and protein source, the isolated species should be capable of degrading and metabolizing some of the wheat bran constituents. Based on the metabolic capacity documented in literature, the isolates likely thrived on proteins and some residual easily fermentable starch in the current set-up, while 
501 in a protein-rich medium, we have previously revealed that the wheat bran-colonizing species

502 possessed arabinoxylan and cellulose degrading enzymatic potential. Combining the new insights

503 from this study with previous observations suggests that adhesion is a rather common trait among

504 gut bacteria, that the outcome of wheat bran colonization is determined by species competition and

505 that external conditions such as $\mathrm{pH}$ and nutrient availability tip the balance in favor of the best-

506 adapted species (Macfarlane et al. 2011). It would, hence, be of interest to modify the experimental

507 conditions, as outlined above, to enrich arabinoxylan and cellulose degrading species. Pure culture

508 studies should then be performed to characterize the fermentation of wheat bran components by

509 the obtained isolates and to further our understanding of the mechanisms of wheat bran attachment

510 which could include Extracellular Polymeric Substances (EPS) or even a cellulosome enzyme

511 system, in case of $R$. champanellensis and common adhesive features such as pili and fimbriae.

512 Finally, the co-culturing of different isolates would be interesting to unravel cooperative and

513 competitive interactions during substrate fermentation.

514

515 ACKNOWLEDGEMENTS

516

517 We would like to acknowledge Veerle Rober for technical assistance and Jolien De Paepe for 518 reviewing this manuscript. 
521

522

523

524

525

526

527

528

529

530

531

532

533

534

535

536

537

538

539

540

541

542

543

544

545

546

547

548

549

550

551

552

553

554

555

556

557

558

559

560

561

562

563

564

565

566

567

\section{References}

Altschul SF, Gish W, Miller W, Myers EW, and Lipman DJ. 1990. Basic Local Alignment Search Tool. Journal of Molecular Biology 215:403-410. DOI 10.1006/jmbi.1990.9999

Amrein TM, Granicher P, Arrigoni E, and Amado R. 2003. In vitro digestibility and colonic fermentability of aleurone isolated from wheat bran. Lebensmittel-Wissenschaft Und-Technologie-Food Science and Technology 36:451-460. 10.1016/S0023-6438(03)00036-7

Araujo JC, Teran FC, Oliveira RA, Nour EAA, Montenegro MAP, Campos JR, and Vazoller RF. 2003. Comparison of hexamethyidisilazane and critical point drying treatments for SEM analysis of anaerobic biofilms and granular sludge. Journal of Electron Microscopy 52:429-433. DOI 10.1093/jmicro/52.4.429

Arte E, Rizzello CG, Verni M, Nordlund E, Katina K, and Coda R. 2015. Impact of Enzymatic and Microbial Bioprocessing on Protein Modification and Nutritional Properties of Wheat Bran. J Agric Food Chem 63:8685-8693. 10.1021/acs.jafc.5b03495

Belzer C, and de Vos WM. 2012. Microbes inside-from diversity to function: the case of Akkermansia. Isme Journal 6:1449-1458. 10.1038/ismej.2012.6

Berger SA, Krompass D, and Stamatakis A. 2011. Performance, accuracy, and Web server for evolutionary placement of short sequence reads under maximum likelihood. Systematic Biology 60:291-302. 10.1093/sysbio/syr010

Bhat P, Albert MJ, Rajan D, Ponniah J, Mathan VI, and Baker SJ. 1980. Bacterial-Flora of the Jejunum - a Comparison of Luminal Aspirate and Mucosal Biopsy. Journal of Medical Microbiology 13:247256.

Bollinger RR, Barbas AS, Bush EL, Lin SS, Lin SS, and Parker W. 2007a. Biofilms in the normal human large bowel: fact rather than fiction. Gut 56:1481-1482.

Bollinger RR, Barbas AS, Bush EL, Lin SS, and Parker W. 2007b. Biofilms in the large bowel suggest an apparent function of the human vermiform appendix. Journal of Theoretical Biology 249:826831. 10.1016/j.jtbi.2007.08.032

Brady CL, Venter SN, Cleenwerck I, Engelbeen K, Vancanneyt M, Swings J, and Coutinho TA. 2009. Pantoea vagans sp nov., Pantoea eucalypti sp nov., Pantoea deleyi sp nov and Pantoea anthophila sp nov. International Journal of Systematic and Evolutionary Microbiology 59:23392345. 10.1099/ijs.0.009241-0

Browne HP, Forster SC, Anonye BO, Kumar N, Neville BA, Stares MD, Goulding D, and Lawley TD. 2016. Culturing of 'unculturable' human microbiota reveals novel taxa and extensive sporulation. Nature 533:543-546. 10.1038/nature17645

Caminero A, Herran AR, Nistal E, Perez-Andres J, Vaquero L, Vivas S, de Morales JMGR, Albillos SM, and Casqueiro J. 2014. Diversity of the cultivable human gut microbiome involved in gluten metabolism: isolation of microorganisms with potential interest for coeliac disease. FEMS Microbiology Ecology 88:309-319. 10.1111/1574-6941.12295

Cantarel BL, Coutinho PM, Rancurel C, Bernard T, Lombard V, and Henrissat B. 2009. The CarbohydrateActive EnZymes database (CAZy): an expert resource for Glycogenomics. Nucleic Acids Research 37:D233-D238. 10.1093/nar/gkn663

Chen W, Zhang CK, Cheng Y, Zhang S, and Zhao H. 2013. A comparison of methods for clustering $16 \mathrm{~S}$ rRNA sequences into OTUs. PLoS One 8:e70837. 10.1371/journal.pone.0070837

Chick H, Cutting ME, Martin C, and Slack E. 1947. Observations on the digestibility and nutritive value of the nitrogenous constituents of wheat bran. Br J Nutr 1:161-182.

Chung WS, Walker AW, Louis P, Parkhill J, Vermeiren J, Bosscher D, Duncan SH, and Flint HJ. 2016. Modulation of the human gut microbiota by dietary fibres occurs at the species level. BMC Biol 14:3. 10.1186/s12915-015-0224-3 
Cole JR, Wang Q, Fish JA, Chai BL, McGarrell DM, Sun YN, Brown CT, Porras-Alfaro A, Kuske CR, and Tiedje JM. 2014. Ribosomal Database Project: data and tools for high throughput rRNA analysis. Nucleic Acids Research 42:D633-D642. 10.1093/nar/gkt1244

Cummings JH, Pomare EW, Branch WJ, Naylor CP, and Macfarlane GT. 1987. Short chain fatty acids in human large intestine, portal, hepatic and venous blood. Gut 28:1221-1227.

De Boever P, Deplancke B, and Verstraete W. 2000. Fermentation by gut microbiota cultured in a simulator of the human intestinal microbial ecosystem is improved by supplementing a soygerm powder. Journal of Nutrition 130:2599-2606.

De Paepe K, Kerckhof F-M, Verspreet J, Courtin CM, and Van de Wiele T. 2017. Inter-individual differences determine the outcome of wheat bran colonization by the human gut microbiome. Environmental Microbiology 19:3251-3267. 10.1111/1462-2920.13819

De Paepe K, Verspreet J, Verbeke K, Raes J, Courtin CM, and Van de Wiele T. 2018. Introducing insoluble wheat bran as a gut microbiota niche in an in vitro dynamic gut model stimulates propionate and butyrate production and induces colon region specific shifts in the luminal and mucosal microbial community. Environmental Microbiology 20:3406-3426. 10.1111/1462-2920.14381

Dornez E, Joye IJ, Gebruers K, Delcour JA, and Courtin CM. 2006. Wheat-kernel-associated endoxylanases consist of a majority of microbial and a minority of wheat endogenous endoxylanases. Journal of Agricultural and Food Chemistry 54:4028-4034. 10.1021/jf060129d

Duncan SH, Hold GL, Harmsen HJM, Stewart CS, and Flint HJ. 2002. Growth requirements and fermentation products of Fusobacterium prausnitzii, and a proposal to reclassify it as Faecalibacterium prausnitzii gen. nov., comb. nov. International Journal of Systematic and Evolutionary Microbiology 52:2141-2146. 10.1099/ijs.0.02241-0

Duncan SH, Louis $\mathrm{P}$, Thomson JM, and Flint HJ. 2009. The role of $\mathrm{pH}$ in determining the species composition of the human colonic microbiota. Environmental Microbiology 11:2112-2122. 10.1111/j.1462-2920.2009.01931.x

Duncan SH, Russell WR, Quartieri A, Rossi M, Parkhill J, Walker AW, and Flint HJ. 2016. Wheat bran promotes enrichment within the human colonic microbiota of butyrate-producing bacteria that release ferulic acid. Environmental Microbiology 18:2214-2225. 10.1111/1462-2920.13158

Foroni E, Serafini F, Amidani D, Turroni F, He F, Bottacini F, Motherway MO, Viappiani A, Zhang ZD, Rivetti C, van Sinderen D, and Ventura M. 2011. Genetic analysis and morphological identification of pilus-like structures in members of the genus Bifidobacterium. Microbial Cell Factories 10. 10.1186/1475-2859-10-S1-S16

Gaastra W, Kusters JG, Van Duijkeren E, and Lipman LA. 2014. Escherichia fergusonii. Veterinary Microbiology 172:7-12. 10.1016/j.vetmic.2014.04.016

Gao D, Luan Y, Wang Q, Liang Q, and Qi Q. 2015. Construction of cellulose-utilizing Escherichia coli based on a secretable cellulase. Microbial Cell Factories 14:159. 10.1186/s12934-015-0349-7

Greub G. 2012. Culturomics: a new approach to study the human microbiome. Clin Microbiol Infect 18:1157-1159. 10.1111/1469-0691.12032

Hall TA. 1999. BioEdit: a user-friendly biological sequence alignment editor and analysis program for Windows 95/98/NT. Nucleic Acids Symposium Series 41:95-98. citeulike-article-id:691774

Hemdane S, Jacobs PJ, Dornez E, Verspreet J, Delcour JA, and Courtin CM. 2016. Wheat (Triticum aestivum L.) Bran in Bread Making: A Critical Review. Comprehensive Reviews in Food Science and Food Safety 15:28-42. 10.1111/1541-4337.12176

Hidalgo-Cantabrana C, Sanchez B, Milani C, Ventura M, Margolles A, and Ruas-Madiedo P. 2014. Genomic Overview and Biological Functions of Exopolysaccharide Biosynthesis in Bifidobacterium spp. Applied and Environmental Microbiology 80:9-18. 10.1128/Aem.02977-13

Holdeman LV, Cato EP, Moore WEC, and University VPIaS. 1977. Anaerobe laboratory manual. Blacksburg, Va.: V.P.I. Anaerobe Laboratory. 
616

617

618

619

620

621

622

623

624

625

626

627

628

629

630

631

632

633

634

635

636

637

638

639

640

641

642

643

644

645

646

647

648

649

650

651

652

653

654

655

656

657

658

659

660

661

662

663

Hugon P, Dufour JC, Colson P, Fournier PE, Sallah K, and Raoult D. 2015. A comprehensive repertoire of prokaryotic species identified in human beings. Lancet Infectious Diseases 15:1211-1219. 10.1016/S1473-3099(15)00293-5

Johansson MEV, Ambort D, Pelaseyed T, Schutte A, Gustafsson JK, Ermund A, Subramani DB, HolmenLarsson JM, Thomsson KA, Bergstrom JH, van der Post S, Rodriguez-Pineiro AM, Sjovall $\mathrm{H}$, Backstrom M, and Hansson GC. 2011. Composition and functional role of the mucus layers in the intestine. Cellular and Molecular Life Sciences 68:3635-3641. 10.1007/s00018-011-0822-3

Kankainen M, Paulin L, Tynkkynen S, von Ossowski I, Reunanen J, Partanen P, Satokari R, Vesterlund S, Hendrickx APA, Lebeer S, De Keersmaecker SCJ, Vanderleyden J, Hamalainen T, Laukkanen S, Salovuori N, Ritari J, Alatalo E, Korpela R, Mattila-Sandholm T, Lassig A, Hatakka K, Kinnunen KT, Karjalainen H, Saxelin M, Laakso K, Surakka A, Palva A, Salusjarvi T, Auvinen P, and de Vos WM. 2009. Comparative genomic analysis of Lactobacillus rhamnosus $G G$ reveals pili containing a human-mucus binding protein. Proceedings of the National Academy of Sciences of the United States of America 106:17193-17198. 10.1073/pnas.0908876106

Katina K, Juvonen R, Laitila A, Flander L, Nordlund E, Kariluoto S, Piironen V, and Poutanen K. 2012. Fermented Wheat Bran as a Functional Ingredient in Baking. Cereal Chemistry 89:126-134. 10.1094/Cchem-08-11-0106

Khan MT, Duncan SH, Stams AJ, van Dijl JM, Flint HJ, and Harmsen HJ. 2012. The gut anaerobe Faecalibacterium prausnitzii uses an extracellular electron shuttle to grow at oxic-anoxic interphases. Isme Journal 6:1578-1585. 10.1038/ismej.2012.5

Khan MT, van Dijl JM, and Harmsen HJM. 2014. Antioxidants Keep the Potentially Probiotic but Highly Oxygen-Sensitive Human Gut Bacterium Faecalibacterium prausnitzii Alive at Ambient Air. PLoS One 9. 10.1371/journal.pone.0096097

Kozich JJ, Westcott SL, Baxter NT, Highlander SK, and Schloss PD. 2013. Development of a Dual-Index Sequencing Strategy and Curation Pipeline for Analyzing Amplicon Sequence Data on the MiSeq Illumina Sequencing Platform. Applied and Environmental Microbiology 79:5112-5120. 10.1128/Aem.01043-13

Lagier JC, Armougom F, Million M, Hugon P, Pagnier I, Robert C, Bittar F, Fournous G, Gimenez G, Maraninchi M, Trape JF, Koonin EV, La Scola B, and Raoult D. 2012. Microbial culturomics: paradigm shift in the human gut microbiome study. Clin Microbiol Infect 18:1185-1193. 10.1111/1469-0691.12023

Lagier JC, Khelaifia S, Alou MT, Ndongo S, Dione N, Hugon P, Caputo A, Cadoret F, Traore SI, Seck EH, Dubourg G, Durand G, Mourembou G, Guilhot E, Togo A, Bellali S, Bachar D, Cassir N, Bittar F, Delerce J, Mailhe M, Ricaboni D, Bilen M, Nieko NPMD, Badiane NMD, Valles C, Mouelhi D, Diop K, Million M, Musso D, Abrahao J, Azhar El, Bibi F, Yasir M, Diallo A, Sokhna C, Djossou F, Vitton V, Robert C, Rolain JM, La Scola B, Fournier PE, Levasseur A, and Raoult D. 2016. Culture of previously uncultured members of the human gut microbiota by culturomics. Nature Microbiology 1. 10.1038/Nmicrobiol.2016.203

Lane. 1991. 16S/23S rRNA sequencing. In: Stackebrandt E, and Goodfellow M, eds. Nucleic acid techniques in bacterial systematics: Wiley, 115-175.

Lanfear R. 2015. sangeranalyseR: sangeranalyseR: a suite of functions for the analysis of Sanger sequence data in R. R package version 0.1.0.

Lawley TD, and Walker AW. 2013. Intestinal colonization resistance. Immunology 138:1-11. 10.1111/j.1365-2567.2012.03616.x

Lebeer S, Claes I, Tytgat HLP, Verhoeven TLA, Marien E, von Ossowski I, Reunanen J, Palva A, de Vos WM, De Keersmaecker SCJ, and Vanderleyden J. 2012. Functional Analysis of Lactobacillus rhamnosus GG Pili in Relation to Adhesion and Immunomodulatory Interactions with Intestinal Epithelial Cells. Applied and Environmental Microbiology 78:185-193. 10.1128/Aem.06192-11

Peer] reviewing PDF | (2018:10:31800:1:1:NEW 14 Dec 2018) 
664

665

666

667

668

669

670

671

672

673

674

675

676

677

678

679

680

681

682

683

684

685

686

687

688

689

690

691

692

693

694

695

696

697

698

699

700

701

702

703

704

705

706

707

708

709

710

711

Leitch ECM, Walker AW, Duncan SH, Holtrop G, and Flint HJ. 2007. Selective colonization of insoluble substrates by human faecal bacteria. Environmental Microbiology 9:667-679. DOI 10.1111/j.1462-2920.2006.01186.x

Letunic I, and Bork P. 2016. Interactive tree of life (iTOL) v3: an online tool for the display and annotation of phylogenetic and other trees. Nucleic Acids Research 44:W242-W245. 10.1093/nar/gkw290

Lombard V, Ramulu HG, Drula E, Coutinho PM, and Henrissat B. 2014. The carbohydrate-active enzymes database (CAZy) in 2013. Nucleic Acids Research 42:D490-D495. 10.1093/nar/gkt1178

Lopez-Siles M, Duncan SH, Garcia-Gil L, and Martinez-Medina M. 2017. Faecalibacterium prausnitzii: from microbiology to diagnostics and prognostics. Isme Journal 11:841-852. 10.1038/ismej.2016.176

Macfarlane GT, Cummings JH, and Allison C. 1986. Protein-Degradation by Human Intestinal Bacteria. Journal of General Microbiology 132:1647-1656.

Macfarlane S, and Dillon JF. 2007. Microbial biofilms in the human gastrointestinal tract. Journal of Applied Microbiology 102:1187-1196. 10.1111/j.1365-2672.2007.03287.x

Macfarlane S, Hopkins MJ, and Macfarlane GT. 2011. Bacterial Growth and Metabolism on Surfaces in the Large Intestine. Microbial Ecology in Health and Disease 12. 10.3402/mehd.v12i2.8103

Macfarlane S, and Macfarlane GT. 2006. Composition and metabolic activities of bacterial biofilms colonizing food residues in the human gut. Appl Environ Microbiol 72:6204-6211. 10.1128/AEM.00754-06

Macfarlane S, McBain AJ, and Macfarlane GT. 1997. Consequences of biofilm and sessile growth in the large intestine. Advances in Dental Research 11:59-68.

Madigan MT, and Brock TD. 2011. Brock Biology of Microorganisms: Pearson.

Martin R, Miquel S, Benevides L, Bridonneau C, Robert V, Hudault S, Chain F, Berteau O, Azevedo V, Chatel JM, Sokol H, Bermudez-Humaran LG, Thomas M, and Langella P. 2017. Functional Characterization of Novel Faecalibacterium prausnitzii Strains Isolated from Healthy Volunteers: A Step Forward in the Use of F. prausnitzii as a Next-Generation Probiotic. Frontiers in Microbiology 8. 10.3389/fmicb.2017.01226

Marzorati M, Vanhoecke B, De Ryck T, Sadabad MS, Pinheiro I, Possemiers S, Van den Abbeele P, Derycke L, Bracke M, Pieters J, Hennebel T, Harmsen HJ, Verstraete W, and Van de Wiele T. 2014. The HMI (TM) module: a new tool to study the Host-Microbiota Interaction in the human gastrointestinal tract in vitro. Bmc Microbiology 14. 10.1186/1471-2180-14-133

Marzorati M, Vilchez-Vargas R, Bussche JV, Truchado P, Jauregui R, El Hage RA, Pieper DH, Vanhaecke L, and Van de Wiele T. 2017. High-fiber and high-protein diets shape different gut microbial communities, which ecologically behave similarly under stress conditions, as shown in a gastrointestinal simulator. Molecular Nutrition \& Food Research 61. 10.1002/mnfr.201600150

Mazur O, and Zimmer J. 2011. Apo- and cellopentaose-bound structures of the bacterial cellulose synthase subunit BcsZ. Journal of Biological Chemistry 286:17601-17606. 10.1074/jbc.M111.227660

McMurdie PJ, and Holmes S. 2014. Waste Not, Want Not: Why Rarefying Microbiome Data Is Inadmissible. Plos Computational Biology 10. 10.1371/journal.pcbi.1003531

Michlmayr H, Hell J, Lorenz C, Bohmdorfer S, Rosenau T, and Kneifel W. 2013. Arabinoxylan oligosaccharide hydrolysis by family 43 and 51 glycosidases from Lactobacillus brevis DSM 20054. Appl Environ Microbiol 79:6747-6754. 10.1128/AEM.02130-13

Nava GM, Friedrichsen HJ, and Stappenbeck TS. 2011. Spatial organization of intestinal microbiota in the mouse ascending colon. Isme Journal 5:627-638. 10.1038/ismej.2010.161

Oksanen J, Blanchet G, Friendly M, Kindt R, Legendre P, McGlinn D, Minchin PR, O'Hara RB, Simpson GL, Solymos P, Stevens H, Szoecs E, and Wagner H. 2016. vegan: Community Ecology Package. $R$ package version 2.4-0. https://CRAN.R-project.org/package=vegan. 
712

713

714

715

716

717

718

719

720

721

722

723

724

725

726

727

728

729

730

731

732

733

734

735

736

737

738

739

740

741

742

743

744

745

746

747

748

749

750

751

752

753

754

755

756

757

758

759

Plugge CM. 2005. Anoxic media design, preparation, and considerations. Environmental Microbiology 397:3-16. 10.1016/S0076-6879(05)97001-8

Probert HM, and Gibson GR. 2002. Bacterial biofilms in the human gastrointestinal tract. Curr Issues Intest Microbiol 3:23-27.

Prückler M, Lorenz C, Endo A, Kraler M, Durrschmid K, Hendriks K, Soares da Silva F, Auterith E, Kneifel W, and Michlmayr H. 2015. Comparison of homo- and heterofermentative lactic acid bacteria for implementation of fermented wheat bran in bread. Food Microbiol 49:211-219. 10.1016/j.fm.2015.02.014

Pruesse E, Peplies J, and Glockner FO. 2012. SINA: accurate high-throughput multiple sequence alignment of ribosomal RNA genes. Bioinformatics 28:1823-1829. 10.1093/bioinformatics/bts252

Quast C, Pruesse E, Yilmaz P, Gerken J, Schweer T, Yarza P, Peplies J, and Glöckner FO. 2013. The SILVA ribosomal RNA gene database project: improved data processing and web-based tools. Nucleic Acids Research 41:D590-D596. 10.1093/nar/gks1219

R Core Team. 2016. R: A language and environment for statistical computing. R Foundation for Statistical Computing, Vienna, Austria. https://www.R-project.org/.

Richardson AJ, McKain N, and Wallace RJ. 2013. Ammonia production by human faecal bacteria, and the enumeration, isolation and characterization of bacteria capable of growth on peptides and amino acids. Bmc Microbiology 13. 10.1186/1471-2180-13-6

Rios-Covian D, Gueimonde M, Duncan SH, Flint HJ, and de los Reyes-Gavilan CG. 2015. Enhanced butyrate formation by cross-feeding between Faecalibacterium prausnitzii and Bifidobacterium adolescentis. Fems Microbiology Letters 362. 10.1093/femsle/fnv176

Robrish SA, Oliver C, and Thompson J. 1991. Sugar Metabolism by Fusobacteria - Regulation of Transport, Phosphorylation, and Polymer Formation by Fusobacterium-Mortiferum Atcc- 25557. Infection and Immunity 59:4547-4554.

Roos S, and Jonsson H. 2002. A high-molecular-mass cell-surface protein from Lactobacillus reuteri 1063 adheres to mucus components. Microbiology-Sgm 148:433-442.

Ruas-Madiedo P, Moreno JA, Salazar N, Delgado S, Mayo B, Margolles A, and de los Reyes-Gavilan CG. 2007. Screening of exopolysaccharide-producing Lactobacillus and Bifidobacterium strains isolated from the human intestinal microbiota. Applied and Environmental Microbiology 73:4385-4388. 10.1128/Aem.02470-06

Salamanca-Cardona L, Ashe CS, Stipanovic AJ, and Nomura CT. 2014. Enhanced production of polyhydroxyalkanoates (PHAs) from beechwood xylan by recombinant Escherichia coli. Appl Microbiol Biotechnol 98:831-842. 10.1007/s00253-013-5398-4

Sanchez JI, Marzorati M, Grootaert C, Baran M, Van Craeyveld V, Courtin CM, Broekaert WF, Delcour JA, Verstraete W, and Van de Wiele T. 2009. Arabinoxylan-oligosaccharides (AXOS) affect the protein/carbohydrate fermentation balance and microbial population dynamics of the Simulator of Human Intestinal Microbial Ecosystem. Microbial Biotechnology 2:101-113. 10.1111/j.17517915.2008.00064.x

Savard P, and Roy D. 2009. Determination of Differentially Expressed Genes Involved in Arabinoxylan Degradation by Bifidobacterium longum NCC2705 Using Real-Time RT-PCR. Probiotics and Antimicrobial Proteins 1:121-129. 10.1007/s12602-009-9015-x

Schloss PD, and Westcott SL. 2011. Assessing and Improving Methods Used in Operational Taxonomic Unit-Based Approaches for $16 \mathrm{~S}$ rRNA Gene Sequence Analysis. Applied and Environmental Microbiology 77:3219-3226. 10.1128/Aem.02810-10

Schloss PD, Westcott SL, Ryabin T, Hall JR, Hartmann M, Hollister EB, Lesniewski RA, Oakley BB, Parks DH, Robinson CJ, Sahl JW, Stres B, Thallinger GG, Van Horn DJ, and Weber CF. 2009. Introducing mothur: Open-Source, Platform-Independent, Community-Supported Software for Describing 
760

761

762

763

764

765

766

767

768

769

770

771

772

773

774

775

776

777

778

779

780

781

782

783

784

785

786

787

788

789

790

791

792

793

794

795

796

797

798

799

800

801

802

803

804

805

806

and Comparing Microbial Communities. Applied and Environmental Microbiology 75:7537-7541. 10.1128/Aem.01541-09

Shewry PR. 2009. Wheat. Journal of Experimental Botany 60:1537-1553. 10.1093/jxb/erp058

Stamatakis A. 2014. RAxML version 8: a tool for phylogenetic analysis and post-analysis of large phylogenies. Bioinformatics 30:1312-1313. 10.1093/bioinformatics/btu033

Stevens BJ, and Selvendran RR. 1988. Changes in composition and structure of wheat bran resulting from the action of human faecal bacteria in vitro. Carbohydr Res 183:311-319.

Swidsinski A, Loening-Baucke V, Verstraelen H, Osowska S, and Doerffel Y. 2008. Biostructure of fecal microbiota in healthy subjects and patients with chronic idiopathic diarrhea. Gastroenterology 135:568-579. 10.1053/j.gastro.2008.04.017

Tannock GW. 2004. A special fondness for lactobacilli. Appl Environ Microbiol 70:3189-3194. 10.1128/AEM.70.6.3189-3194.2004

The HC, Thanh DP, Holt KE, Thomson NR, and Baker S. 2016. The genomic signatures of Shigella evolution, adaptation and geographical spread. Nature Reviews Microbiology 14:235-250. 10.1038/nrmicro.2016.10

Thompson J, Robrish SA, Bouma CL, Freedberg DI, and Folk JE. 1997. Phospho-beta-glucosidase from Fusobacterium mortiferum: purification, cloning, and inactivation by 6-phosphoglucono-deltalactone. Journal of Bacteriology 179:1636-1645.

United States Department of Agriculture (USDA). 2016. National Nutrient Database for Standard Reference Release 28.

Van den Abbeele P, Belzer C, Goossens M, Kleerebezem M, De Vos WM, Thas O, De Weirdt R, Kerckhof FM, and Van de Wiele T. 2013. Butyrate-producing Clostridium cluster XIVa species specifically colonize mucins in an in vitro gut model. Isme Journal 7:949-961. 10.1038/ismej.2012.158

Van den Abbeele P, Grootaert C, Possemiers S, Verstraete W, Verbeken K, and Van de Wiele T. 2009. In vitro model to study the modulation of the mucin-adhered bacterial community. Applied Microbiology and Biotechnology 83:349-359. 10.1007/s00253-009-1947-2

Van den Abbeele P, Roos S, Eeckhaut V, MacKenzie DA, Derde M, Verstraete W, Marzorati M, Possemiers S, Vanhoecke B, Van Immerseel F, and Van de Wiele T. 2012. Incorporating a mucosal environment in a dynamic gut model results in a more representative colonization by lactobacilli. Microbial Biotechnology 5:106-115. 10.1111/j.1751-7915.2011.00308.x

van den Broek LAM, Hinz SWA, Beldman G, Vincken JP, and Voragen AGJ. 2008. Bifidobacterium carbohydrases-their role in breakdown and synthesis of (potential) prebiotics. Molecular Nutrition \& Food Research 52:146-163. 10.1002/mnfr.200700121

Walker AW, Duncan SH, Harmsen HJM, Holtrop G, Welling GW, and Flint HJ. 2008. The species composition of the human intestinal microbiota differs between particle-associated and liquid phase communities. Environmental Microbiology 10:3275-3283. DOI 10.1111/j.14622920.2008.01717.x

Walker AW, Duncan SH, McWilliam Leitch EC, Child MW, and Flint HJ. 2005. pH and peptide supply can radically alter bacterial populations and short-chain fatty acid ratios within microbial communities from the human colon. Appl Environ Microbiol 71:3692-3700. 10.1128/AEM.71.7.3692-3700.2005

Wang Q, Garrity GM, Tiedje JM, and Cole JR. 2007. Naive Bayesian classifier for rapid assignment of rRNA sequences into the new bacterial taxonomy. Applied and Environmental Microbiology 73:5261-5267. 10.1128/Aem.00062-07

Wang XY, Cai YP, Sun YJ, Knight R, and Mai V. 2012. Secondary structure information does not improve OTU assignment for partial 16s rRNA sequences. Isme Journal 6:1277-1280.

10.1038/ismej.2011.187

PeerJ reviewing PDF | (2018:10:31800:1:1:NEW 14 Dec 2018) 


\section{Table $\mathbf{1}$ (on next page)}

RDP Seqmatch and NCBI BLAST results from the single colony isolates.

The best hits, with the highest percentage identity (NCBI) and similarity score (S_ab in RDP) are shown. Species obtained by direct plating are indicated in boldface. 


\section{Donor 1}

83,95

78

$\mathbf{8 4 , 8 7}$

79,85

86

76

$\mathbf{8 0 , 8 8 , 9 0}$

$\mathbf{8 2 , 9 1 , 9 2 , 9 3 , 9 4 , 9 6 , 9 7 , 9 9}$

81

$103,105,106,107,108,109,110$

$101,102,104$
Bacillus anthracis/toyonensis/cereus/thuringiensis/

pseudomycoides/mycoides/weihenstephanensis/

marcorestinctum/bingmayongensis/manliponensis/

gaemokensis/cytotoxicus

Bacteroides eggerthii

Bacteroides dorei

Bacteroides fragilis

Bacteroides thetaiotaomicron/faecis

Bifidobacterium bifidum

Bifidobacterium adolescentis/faecale/ruminantium

Collinsella aerofaciens

Dorea formicigenerans

Lactobacillus delbrueckii

Lactobacillus fermentum

\section{Donor 2}

$19,25,28$

Bifidobacterium faecale/adolescentis

21

Bifidobacterium bifidum

26

Bifidobacterium pseudocatenulatum

34

Bifidobacterium faecale/adolescentis/ruminantium

$\mathbf{1 0 , 2 7 , 3 0 , 3 1 , 3 2 , 3 5 , 3 7}$
Escherichia coli/fergusonii/marmotae/vulneris/albertii

Shigella sonnei/flexneri/dysenteriae/boydii 
29

$36,40,41,42,43,44,45,46$

$13,15,18$

153

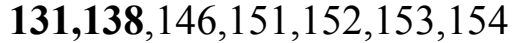

$, 156,157,158,159,160,161$

$143,144,149$

$\mathbf{1 2 8 , 1 2 9 , 1 4 1 , 1 4 5 , 1 4 7}$

$133,137,140$

136,155
Enterococcus durans

Pediococcus pentosaceus

Streptococcus pasteurianus/macedonicus/equinus/

gallolyticus/lutetiensis/loxodontisalivarius/

infantarius/saliviloxodontae

\section{Donor 3}

Enterococcus faecium

Enterococcus faecium/lactis/durans/villorum/hirae/

thailandicus/mundtii/dispar/canintestini/ratti/raffinosus/

pseudoavium/casseliflavus/avium/viikkiensis/gilvus/

malodoratus/devriesei/gallinarum/xiangfangensis/canis/

asini/massiliensis/pallens

Shigella sonnei/flexneri

Escherichia fergusonii

Klebsiella michiganensis/oxytoca/pneumoniae/

quasipneumoniae, Enterobacter cloacae/bugandensis/

cancerogenus/asburiae/xiangfangensis

Escherichia vulneris, Yokenella regensburgei

Kluyvera cryocrescens

Raoultella ornithinolytica/terrigena

Streptococcus lutetiensis/infantarius/equinus

pasteurianus/macedonicus

Pediococcus pentosaceus/stilesii/ claussenii 


\section{Donor 4}

184,185

186

206

$\mathbf{1 8 1 , 1 8 2 , 1 9 5 , 1 9 6 , 2 0 1 , 2 0 2}$

200

187,188,189,190,191,192,193,194 Escherichia coli/fergusonii/vulneris/albertii/marmotae

Shigella flexneri/sonnei/boydii/dysenteriae

Brenneria alni

198
Bacteroides ovatus $(90 \%, 96 \%$ similarity)

Bacteroides ovatus

Bifidobacterium adolescentis/faecale

Collinsella aerofaciens

Eubacterium rectale

1 


\section{Figure 1}

Overview of the experimental set-up.

In this study, wheat bran-metabolizing and colonizing species were isolated from human fecal microbiota obtained from four healthy individuals. To this end, insoluble wheat bran particles with a characterized chemical composition were incorporated in solid agar plates used in a direct plating method on the one hand and added to a liquid broth used in an enrichment procedure on the other hand. As described in details in the materials and methods section of the manuscript, the fecal sample is directly plated onto the solid wheat bran agar plates to isolate wheat bran metabolizing species. Whereas, the enrichment method selects for wheat bran-attaching and metabolizing species by subculturing the wheat bran residue during three consecutive transfers.

The milling of wheat kernels separates the outer tissue layers, collectively termed wheat bran, from the wheat endosperm and germ

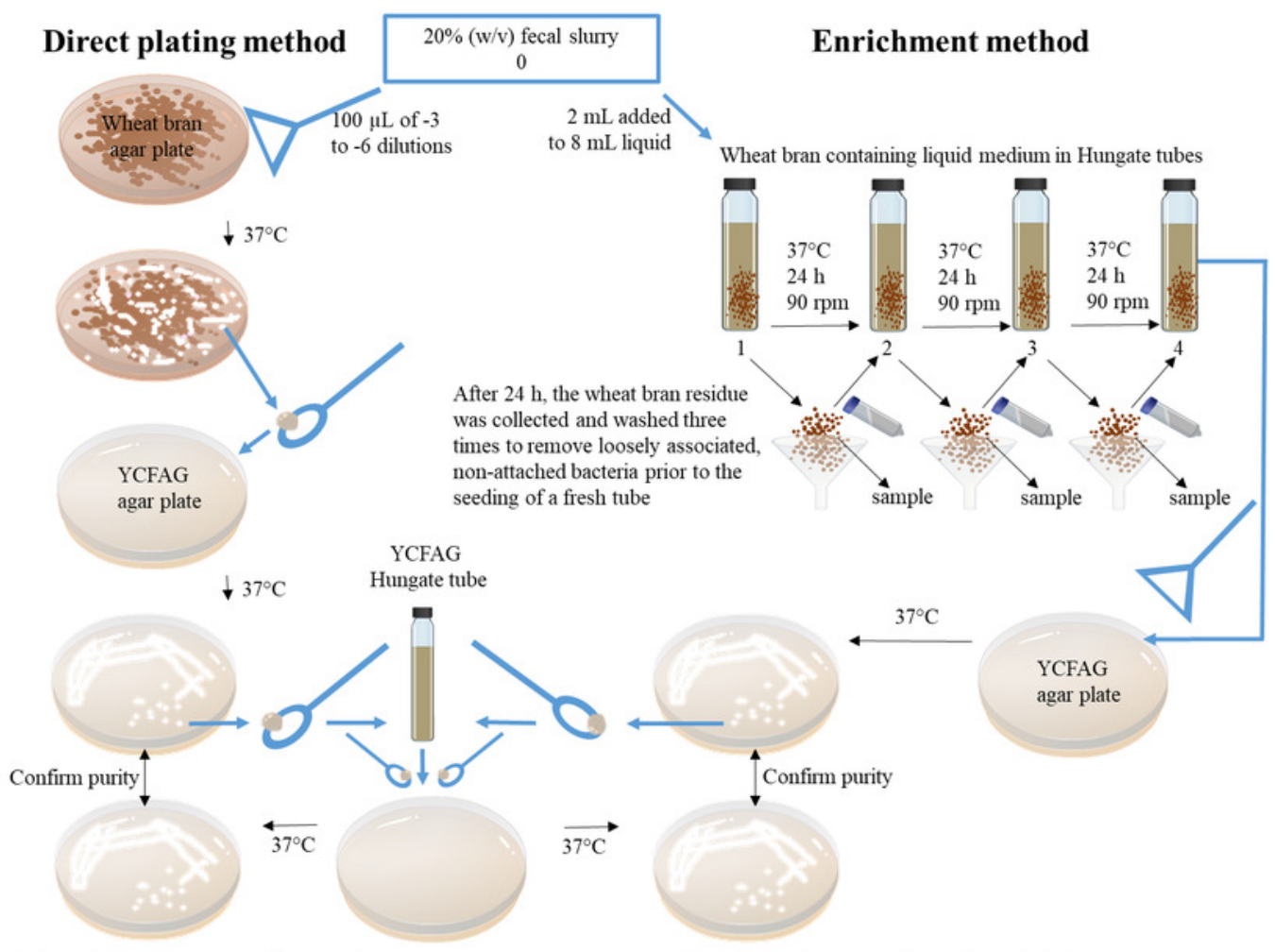




\section{Figure 2}

Short Chain Fatty Acid (SCFA) production by the fecal microbiota derived from four different donors during enrichment with wheat bran as the sole nutrient source.

The fecal sample (enrichment step 0) was incubated with wheat bran for $24 \mathrm{~h}$ (enrichment step 1), after which the wheat bran residue was washed to remove loosely attached bacteria and used to seed a new incubation (enrichment step 2). This procedure was repeated two more times (enrichment step 3 and 4). The $\mathrm{pH}$ is indicated on top of the stacked bars and decreased considerably compared to the starting pH (5.8 and 6.8). 

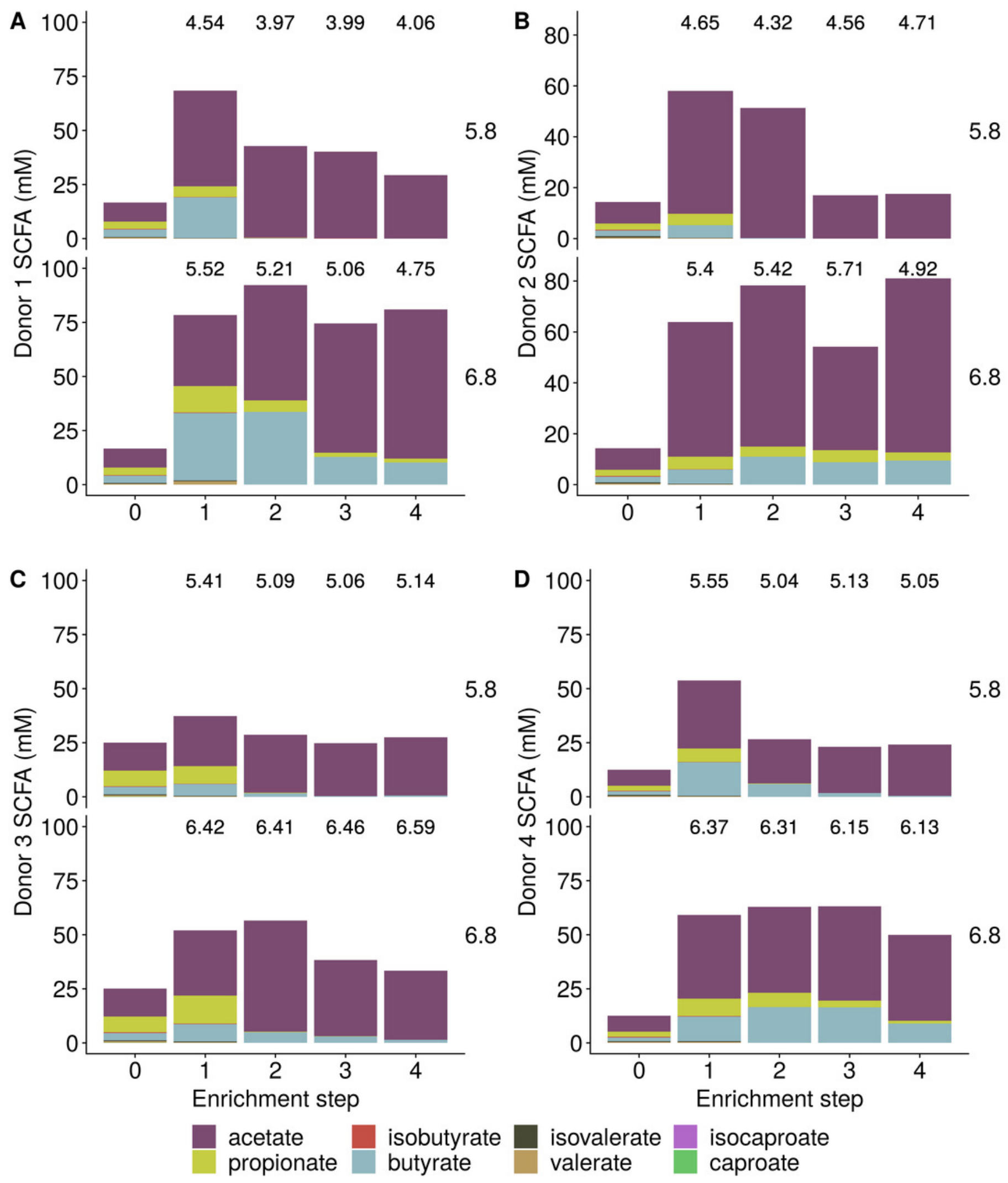


\section{Figure 3}

Reduction in the microbial community richness (Chao 1 richness estimator) and diversity (Shannon diversity index) during consecutive enrichment steps with wheat bran as the sole nutrient source, as shown for donor 1 .

The fecal sample (enrichment step 0) was incubated with wheat bran for $24 \mathrm{~h}$ (enrichment step 1), after which the wheat bran residue was washed to remove loosely attached bacteria and used to seed a new incubation (enrichment step 2). This procedure was repeated two more times (enrichment step 3 and 4). 

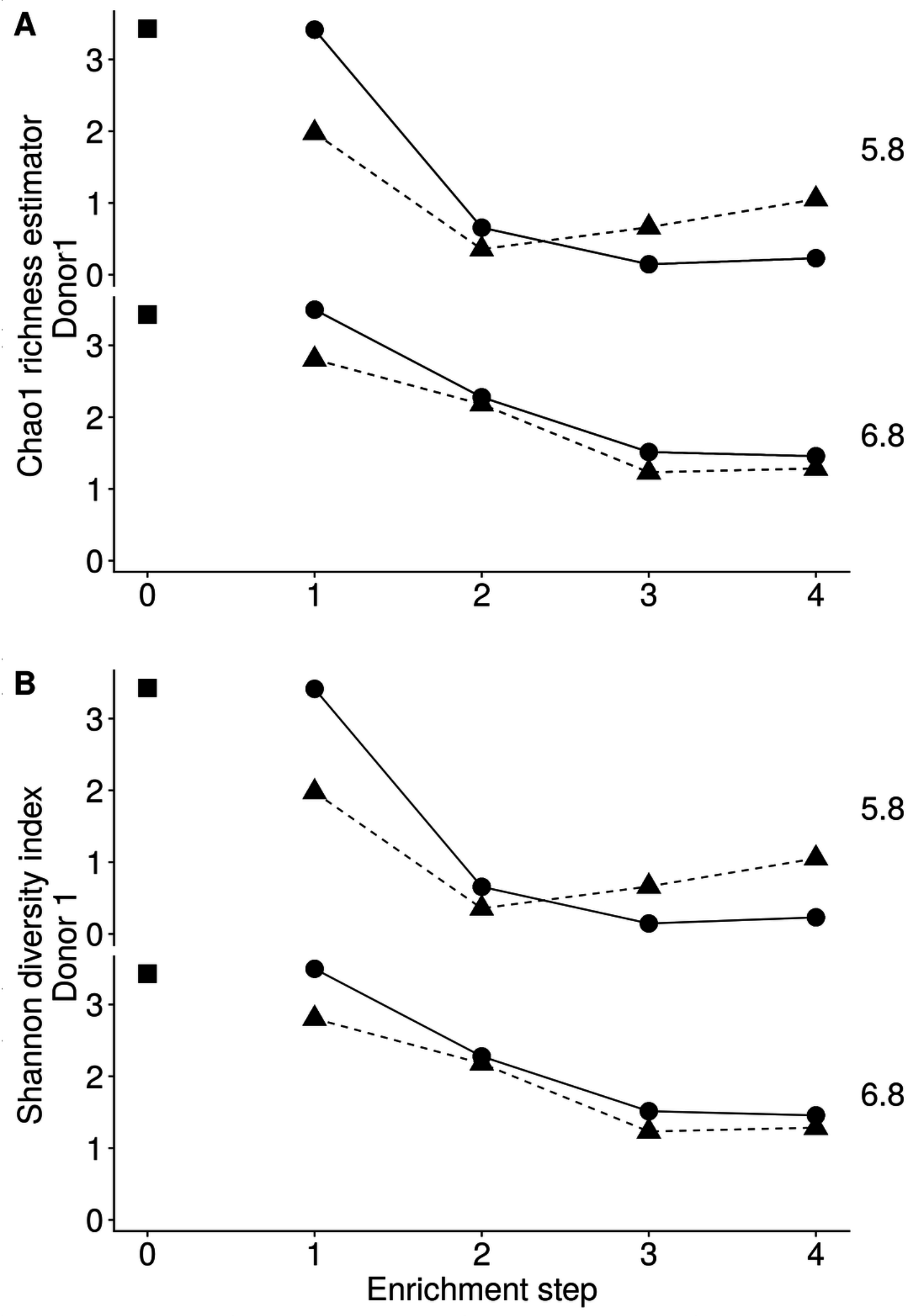


\section{Figure 4}

Shifts in genus (A) and species (B) level microbial community composition of donor 1 during consecutive enrichment steps with wheat bran as the sole nutrient source.

The fecal sample (FS; enrichment step 0) was incubated with wheat bran for $24 \mathrm{~h}$ (enrichment step 1), after which the wheat bran residue was washed to remove loosely attached bacteria and used to seed a new incubation (enrichment step 2). This procedure was repeated two more times (enrichment step 3 and 4). Family level taxa appearing in the genus level plots should be interpreted as unclassified genus belonging to the respective family. 


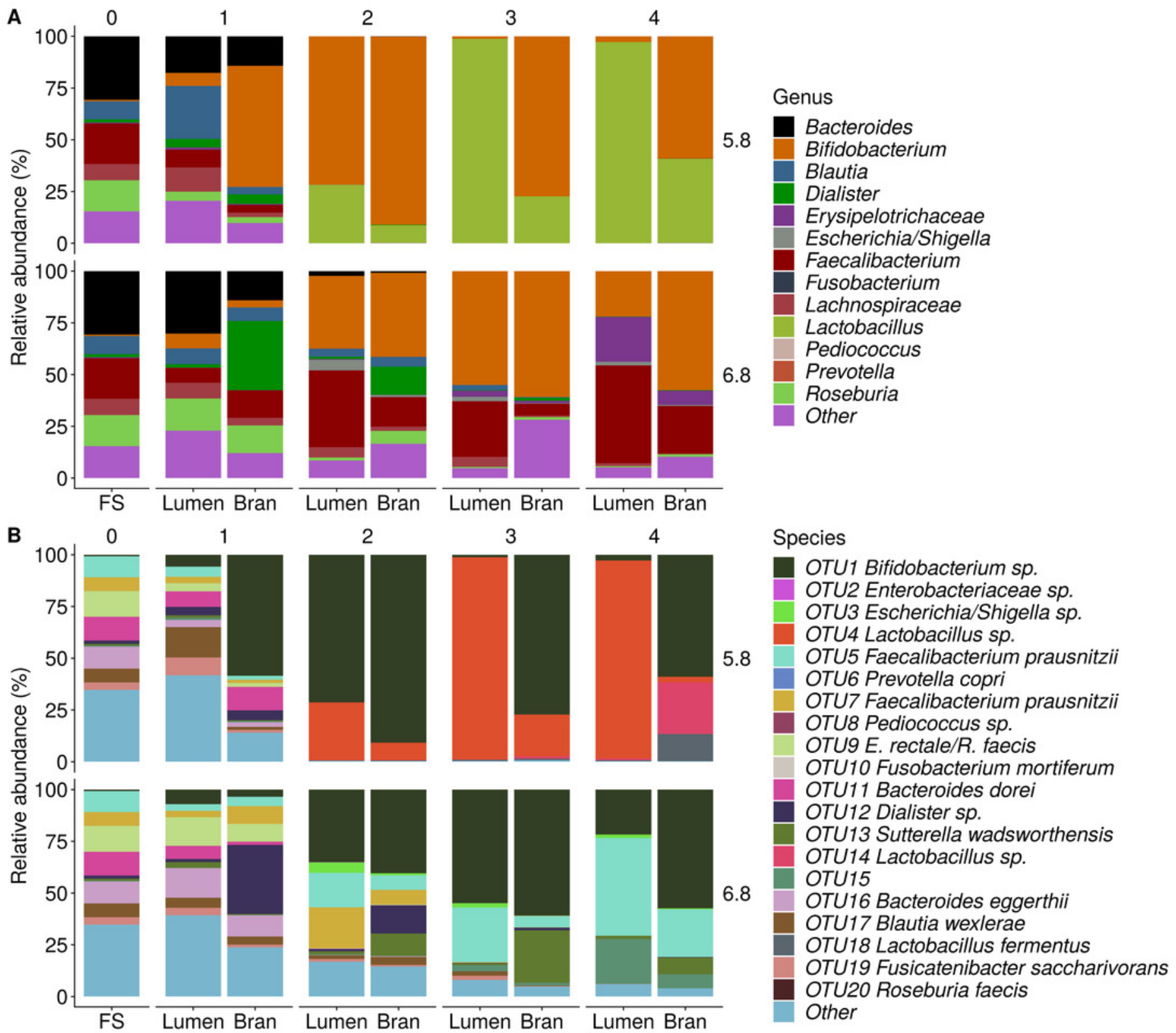




\section{Figure 5}

Shifts in genus (A) and species (B) level microbial community composition of donor 2 and 3 after four enrichment steps with wheat bran as the sole nutrient source.

The fecal sample (FS; enrichment step 0) was incubated with wheat bran for $24 \mathrm{~h}$ (enrichment step 1), after which the wheat bran residue was washed to remove loosely attached bacteria and used to seed a new incubation (enrichment step 2). This procedure was repeated two more times (enrichment step 3 and 4). Only the final enrichment step and FS are shown in this plot. Family level taxa appearing in the genus level plots should be interpreted as unclassified genus belonging to the respective family. 


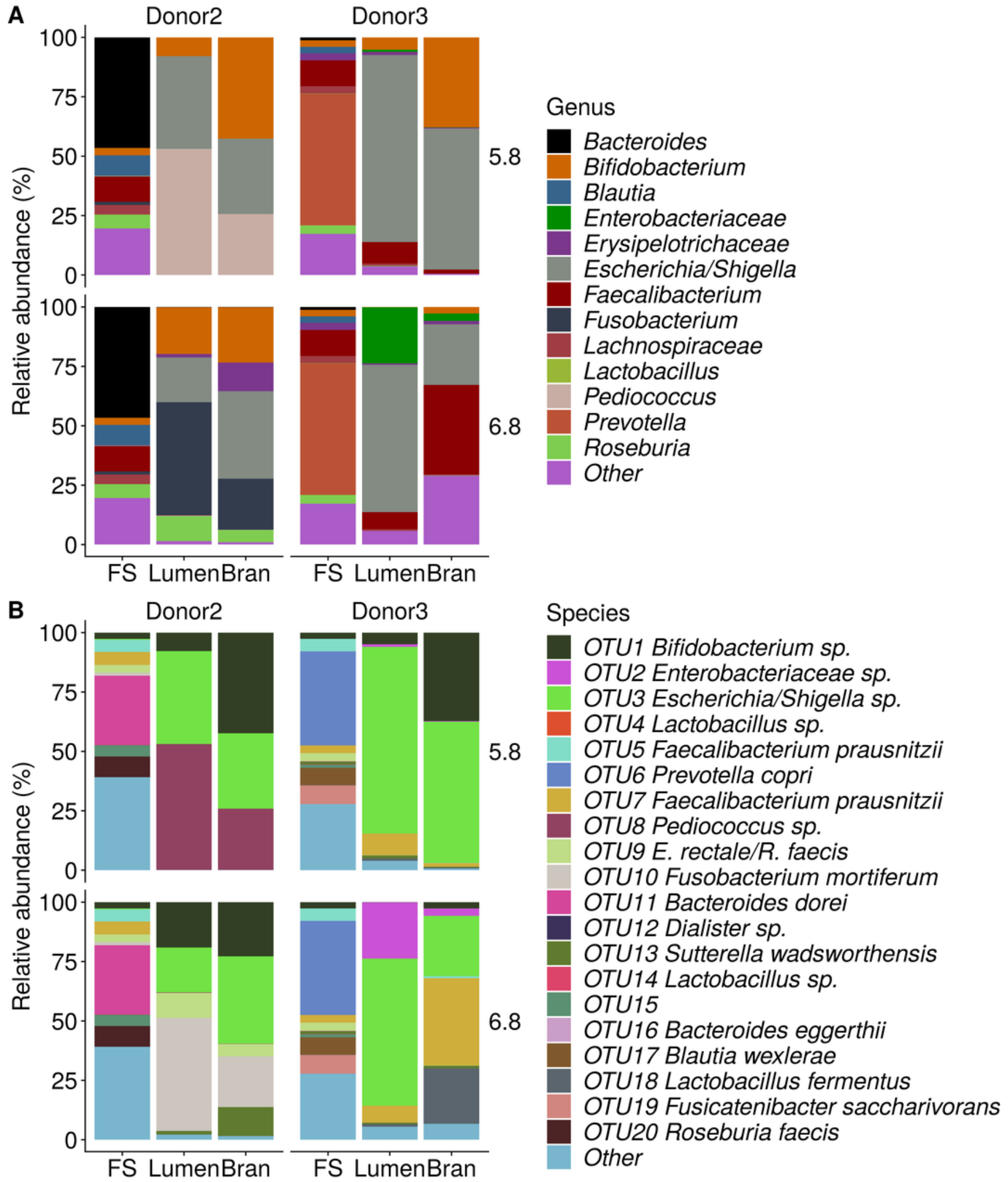




\section{Figure 6}

Genus (A) and species (B) level microbial community composition of donor 4 after the final enrichment step with wheat bran as the sole nutrient source.

The fecal sample (FS; enrichment step 0) was incubated with wheat bran for $24 \mathrm{~h}$ (enrichment step 1), after which the wheat bran residue was washed to remove loosely attached bacteria and used to seed a new incubation (enrichment step 2). This procedure was repeated two more times (enrichment step 3 and 4). Only the final enrichment step was shown in this plot, due to a low read number ( $<100$ reads) in the fecal sample of donor 4. Family level taxa appearing in the genus level plots should be interpreted as unclassified genus belonging to the respective family. 


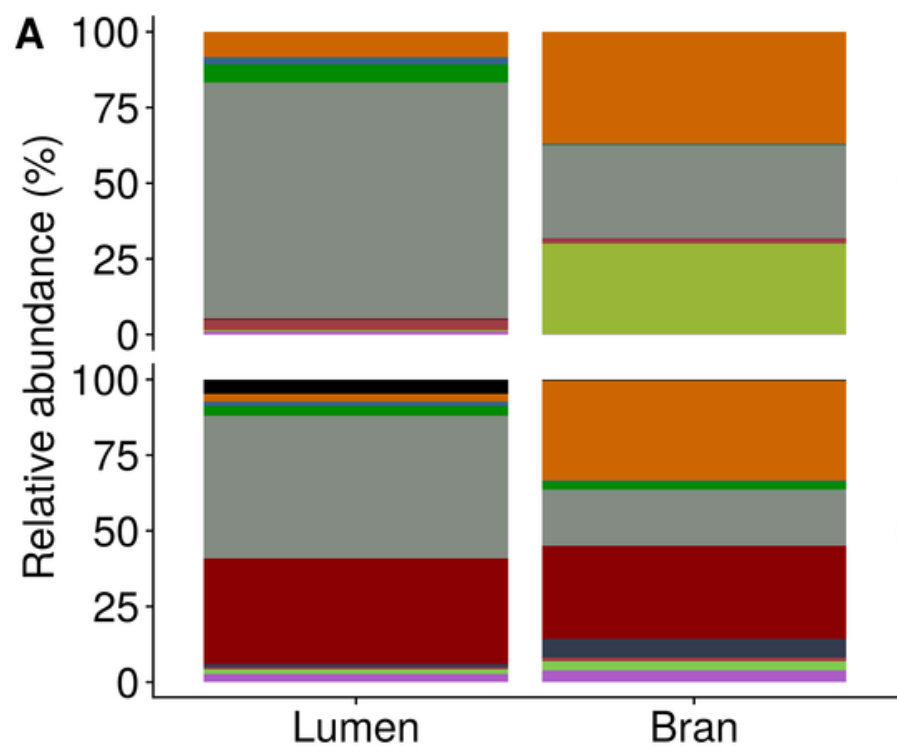
Genus

\section{Acetobacter}
Bacteroides
5.8
Bifidobacterium
Clostridium_XIVa
Clostridium_XVIII
Escherichia/Shigella
Faecalibacterium
Fusicatenibacter
Lachnospiraceae
6.8
Lactobacillus
Roseburia
Sphingomonas
Veillonella
Other

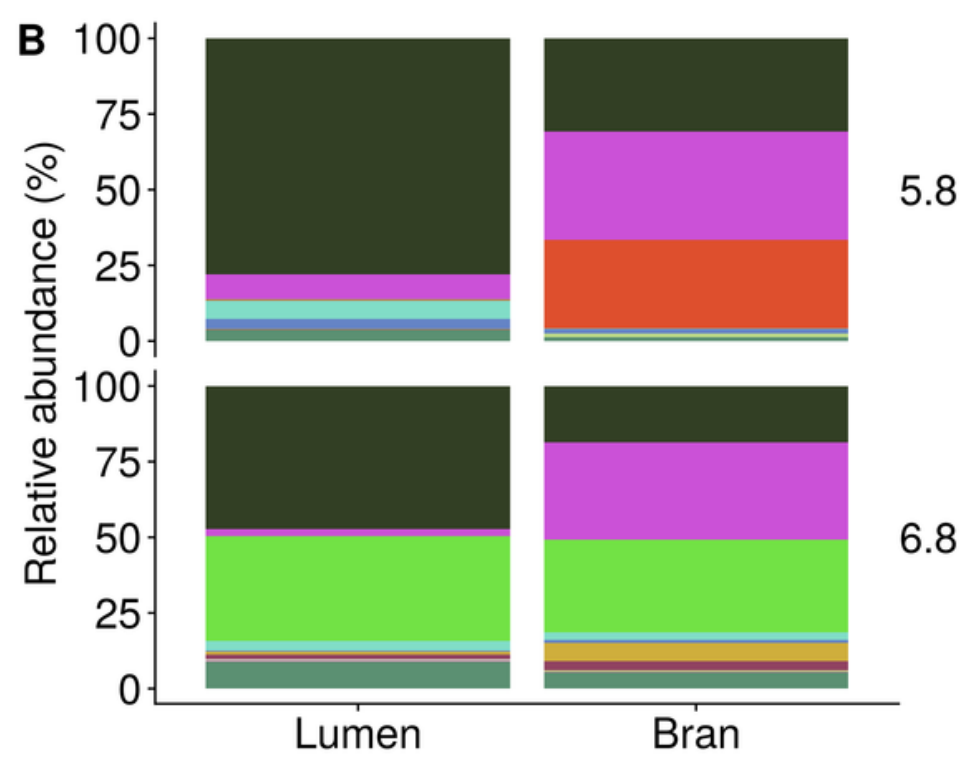

Species

OTU1 Escherichia/Shigella sp.

OTU2 Bifidobacterium sp.

OTU3 Faecalibacterium prausnitzii

OTU4 Lactobacillus gasseri

OTU5 Clostridium XVII

OTU6 Lachnospiraceae sp.

OTU7 Fusicatenibacter saccharivorans

OTU8 Clostridium XIVa sp.

OTU9 Collinsella aerofaciens

OTU10 Eubacterium rectale

6.8

OTU11 Bacteroides vulgatus

OTU12 Bifidobacterium longum

OTU13 Gemmiger formicilis

OTU14 Dialister invisus

Other 


\section{Figure 7}

Removal of starch during pre-digestion.

'(A) Cryo-SEM image of an unmodified wheat bran particle with endosperm starch granules, resulting from the crude milling process, covering the surface of aleurone cells. (B) SEM image of a micronized wheat bran fragment after pre-digestion, without attached starch granules.'
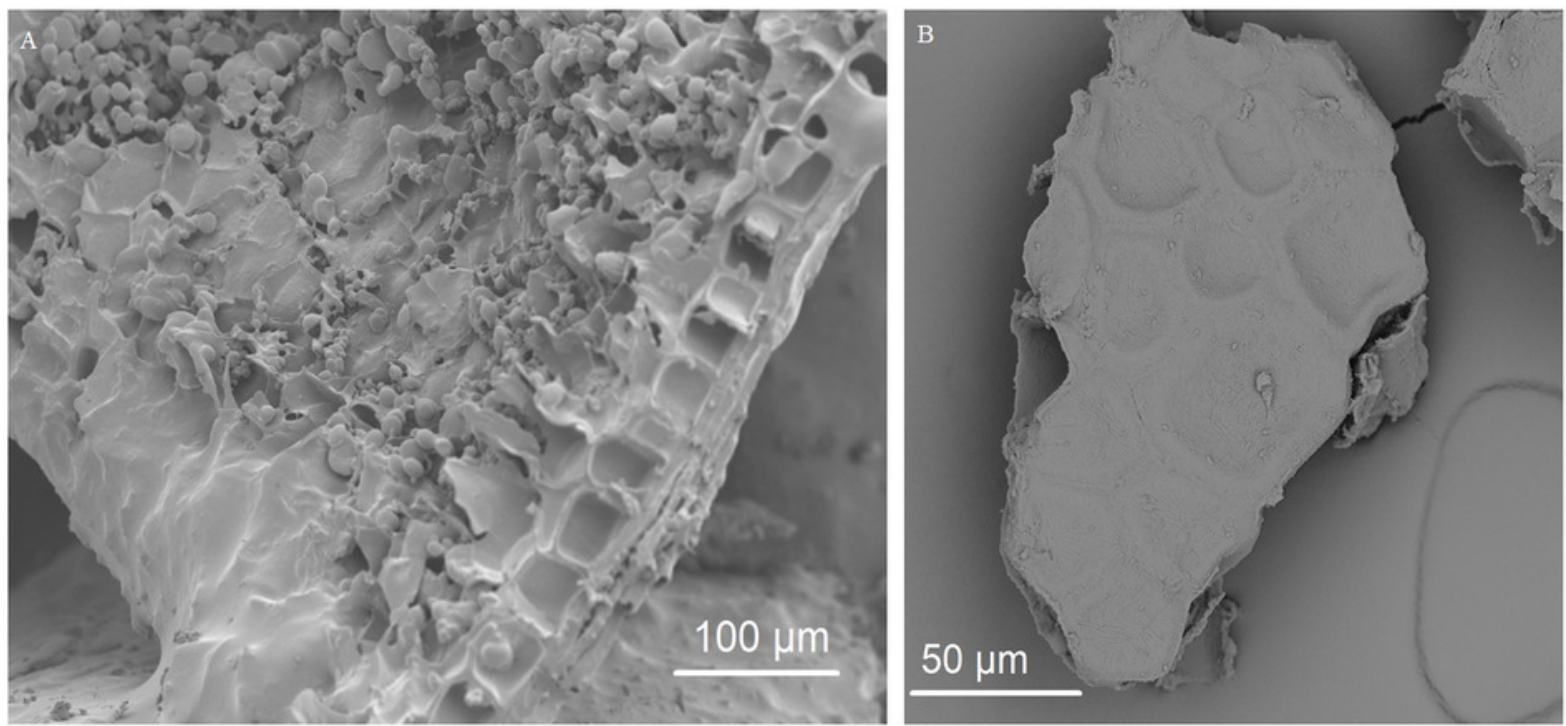


\section{Figure 8}

SEM images of the fermented wheat bran residue after $24 \mathrm{~h}$.

'(A) Uncolonized pericarp tissue. (B) Residual starch granule. (C) Bacteria inside a wheat aleurone cell. (D) Bacteria on aleurone fragment. (E) Partial degradation of aleurone cell. (F) Ligature at the junction of aleurone cells.'

*Note: Auto Gamma Correction was used for the image. This only affects the reviewing manuscript. See original source image if needed for review. 

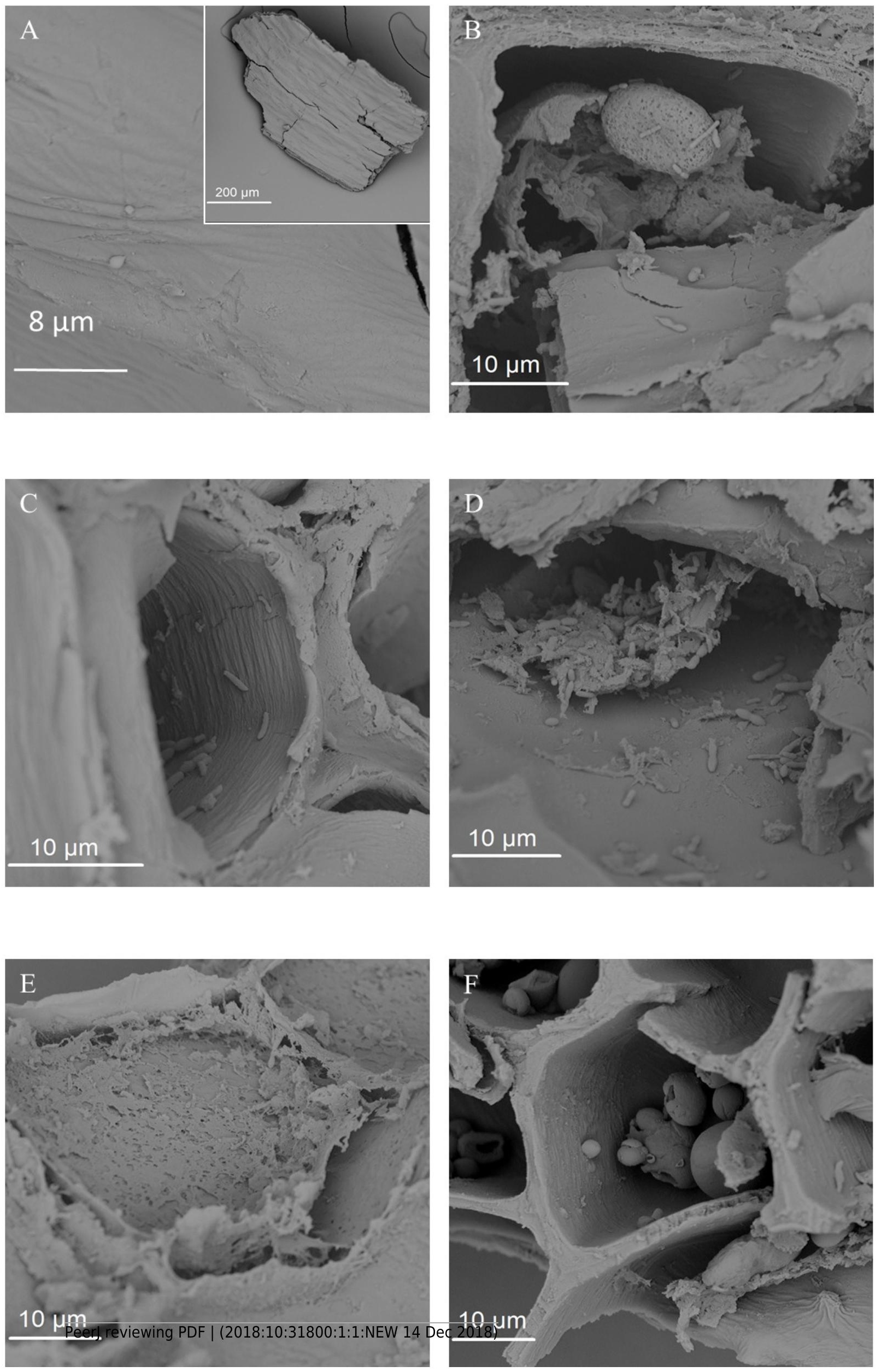